\title{
A CASE STUDY INTO THE ENVIRONMENTAL CONTEXT OF WALKING SCHOOL BUSES AND OTHER MODES OF TRAVEL USED FOR THE JOURNEY TO SCHOOL
}

\author{
by \\ John Forne
}

Thesis

ENVIRONMENTAL STUDIES 593

2004

A 60-point thesis submitted to the Institute of Geography, Victoria University of Wellington, As partial fulfilment for the degree of Masters of Environmental Studies

Institute of Geography, School of Earth Sciences, Victoria University of Wellington, 28 February 2004 


\section{A CASE STUDY INTO THE ENVIRONMENTAL CONTEXT OF WALKING SCHOOL BUSES AND OTHER MODES OF TRAVEL USED FOR THE JOURNEY TO SCHOOL}

John Forne

\section{ABSTRACT}

The journey to school involves a number of different modes of travel. Approximately half of all journeys to school in New Zealand rely on the use of an automobile. This heavy dependence on automobiles involves a number of environmental detractions. Walking School Buses provide an alternative to cars as a mode of travel for the journey to school. This mode of travel compares favourably in a number of respects when compared to other modes of travel and is generating considerable interest.

Based on a case study involving in-depth interviews with ten caregivers this paper compares whether and how Walking School Buses emerged from a different social and cultural background when compared to other modes of travel. Four key influences, in particular parenting culture, the work commitments that caregivers have, the risks posed by strangers and traffic, and the social fragmentation of neighbourhoods were found to be significant in shaping the journey to school. Based on finding a number of similarities between different modes of travel to school it is concluded that the journey to school is embedded in a wider system of social and cultural ideas that shape Walking School Buses and other modes of travel alike.

Key words: journey to school, social and cultural system, Walking School Buses, work commitments, parenting culture, risk, and social fragmentation. 


\section{Acknowledgements}

I thank all the people who contributed to making this paper happen and express my gratitude to have had the opportunity to pursue such a journey. In particular I thank the caregivers who impressed me with their enthusiasm and willingness to participate and without whom this work would not have been. Further, the support of the principals I worked with was invaluable. Thankfully these gatekeepers let me through and were kind to me by assisting bridge the gap with the caregivers. Within the Institution I thank Richard Willis, my supervisor, for accommodating me in shaping this project as one that interests me and thanks to Laurie Jackson for all the feedback on the last minute (almost) drafts. Also a huge thank you goes to James Urry, from the Department of Anthropology who generously gave me many hours of amazing expertise in the discussion of different ways of observing others and ourselves. His experience and passion for helping others develop an acutely perceptive and considered view of the world around us, is inspiring and indelible. Last, but very much not least, thank you Arnica... for everything. 


\title{
Table of Contents
}

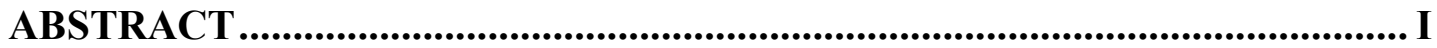

ACKNOWLEDGEMENTS ..........................................................................II

TABLE OF CONTENTS ........................................................................ III

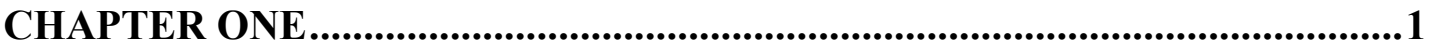

INTRODUCTION $\sim$ SETTING THE SCENE IN WHICH THE JOURNEY BEGINS ................ 1

A REVIEW OF THE WALKING SchOOL Bus PHENOMENON ..................................... 3

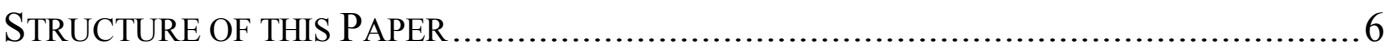

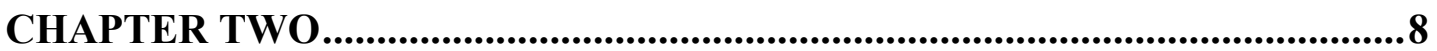

THE JOURNEY TO SCHOOL ENVIRONMENT THROUGH A SOCIAL AND CULTURAL

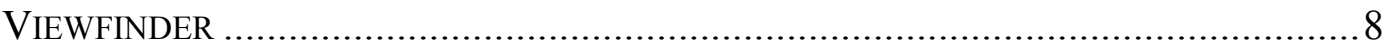

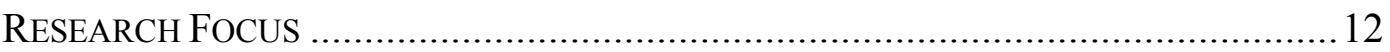

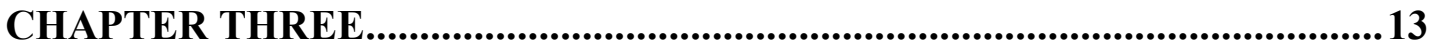

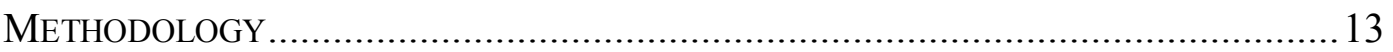

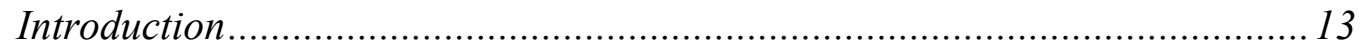

Interviews Involve Normalised Responses........................................... 13

Reflecting on the Limitations of Interviewing ........................................... 14

What does Research Mean Anyway? ............................................................ 14

Recognising the Subjectivity of Research ................................................ 15

Limits to Subjectivity............................................................................ 16

The Importance of Reflecting About Research........................................ 16

Avoiding too Much Reflection ...................................................................... 16

On the Practice of Semi-Structured Interviewing ....................................... 17

Interviewing with an Aide Memoir ........................................................... 17

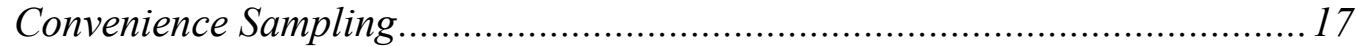

Interviewing two Groups of Caregivers............................................... 18

Interviewing ten Caregivers ...................................................................... 19

Sampling from two Schools - Variation and Bias....................................... 19

Selecting Caregivers ............................................................................ 20

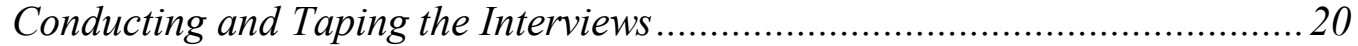

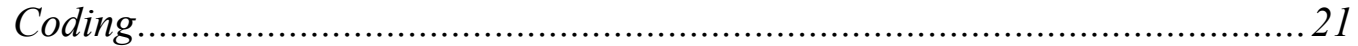

Feedback from Coding on the Interview Technique...................................... 21

Conversation Analysis .............................................................................. 23

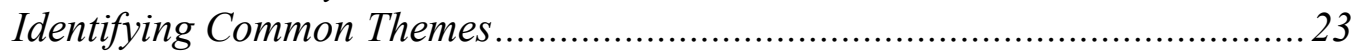

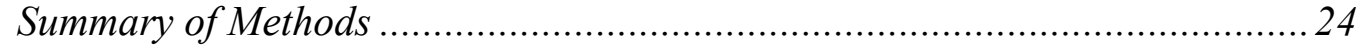

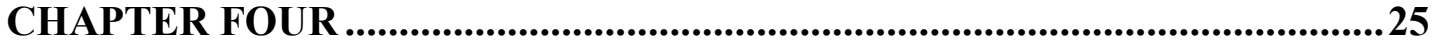

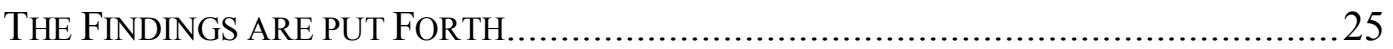

Introduction....................................................................................... 25

Caregiver Ideas About Their Children Being Outdoors................................ 26 
Caregivers on the Stranger Danger Issue ..................................................26

Supervision and Community Building in Response to Stranger Danger...........22

An Opportunity for Children to Develop Healthy Habits................................2 27

Caregiver Ideas About Their Children Developing Independence ................... 28

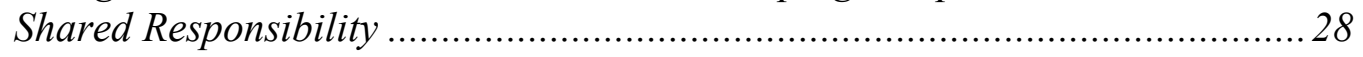

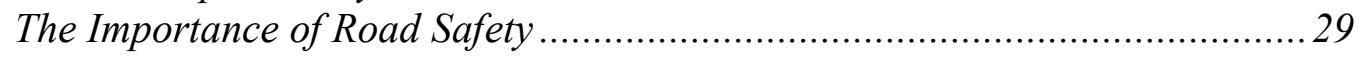

The Availability of Alternative Modes of Travel ...........................................29

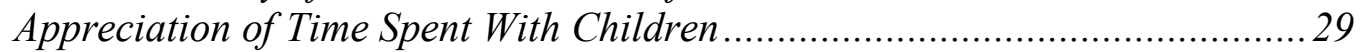

Role Models Provided by Caregivers ............................................................. 30

How did Caregivers get to School When They Were Children? ....................... 30

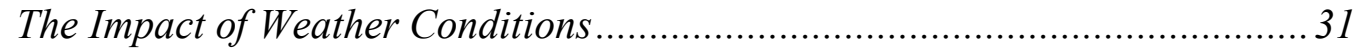

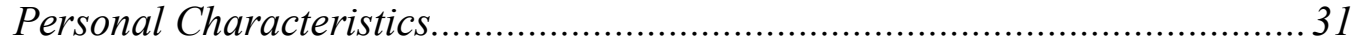

How far is too far-a Comparison of Distance to School.................................. 32

A Deliberate Decision and the Mode of Travel Used.....................................32

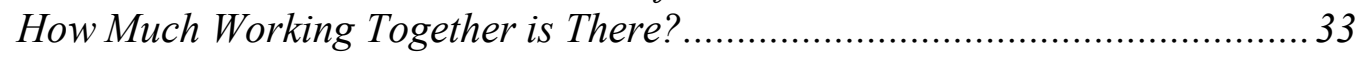

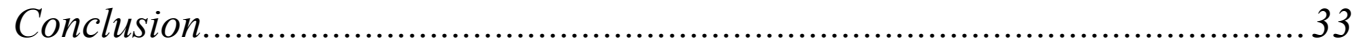

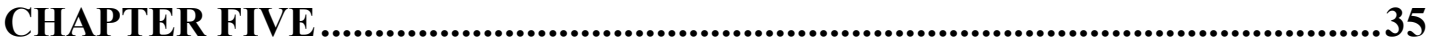

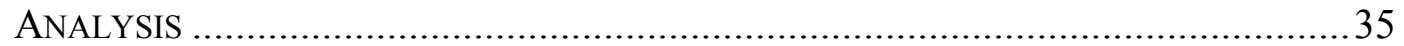

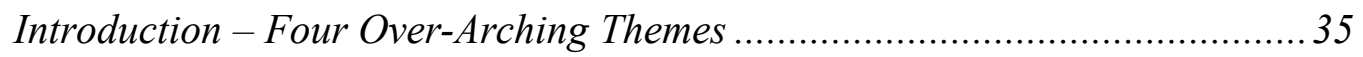

The Pervasiveness of Parenting Culture.......................................................... 36

The Role of Work in Caregivers' Lives............................................................ 37

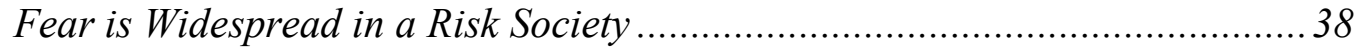

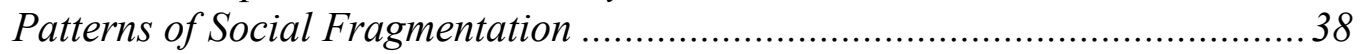

Social and Cultural Influences on Caregivers can be in Conflict......................39

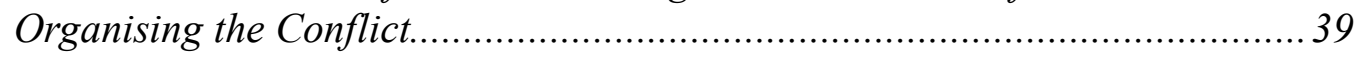

Innovation is Possible ........................................................................ 40

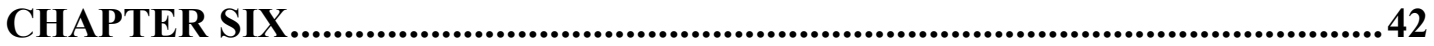

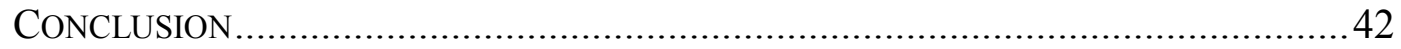

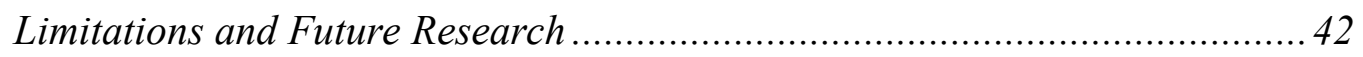

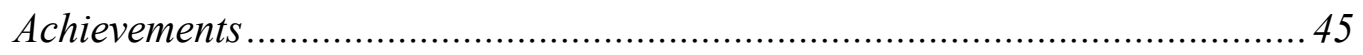

REFERENCES ................................................................................................48

APPENDIX ONE

AIDE MEMOIR

APPENDIX TWO

CODED INTERVIEW DATA 


\section{Chapter One}

\section{Introduction Setting the Scene in Which the Journey Begins}

Since about 1990 there has been a two hundred percent increase in the reliance on automobiles for the journey to school in New Zealand (O'Fallon, 2003). Following this increase around half of New Zealand's primary school children are taken between home and school by car (O'Fallon, 2001). A recent report by the Auckland Regional Council suggests that up to forty percent of Auckland's morning traffic is involved with educational travel (Baalbergen, 2004). The high levels of reliance on automobiles for the journey to school has a number of environmental impacts, including environmental pollution, increased reliance on resource intensive activities, road safety issues, and degradation of community living spaces (Engwicht, 1993). A heavy reliance on automobiles makes neighbourhoods less attractive and vibrant places for people to live. The heartbeat of cities is described by Engwicht (1993) as an exchange- of goods and ideas and the streets provide the veins in which this trade occurs. People who use cars are isolated from interacting with other people in the street and consequently the vibrancy of car-dominated neighbourhoods is diminished.

There are many different modes of travel used for the journey to school. In particular, Walking School Buses (WSBs) provide one of these alternatives. Mouchel (2003) provides a useful introductory description of WSBs as involving adult volunteers walking with small groups of children between their home and school along set routes. As a mode of getting to school, WSBs are described by Kearns, Collins, and Neuwelt (2003) as a structured means of travel as children are supervised by adult-imposed rules. WSB schemes have been linked to a number of environmentally friendly impacts. For example, O'Fallon (2001) finds that WSBs go someway to address the concerns surrounding the journey to school, such as congestion, pollution, and safety. 
Using the journey to school as a window, this paper seeks to develop an understanding of the social and cultural context of environmental issues. For example, Britton (2001) suggests that the mode of travel used for the journey to school provides an illuminating microcosm into pertinent issues, including "citizen responsibility, concern for our children's full personal and social development, and of our ability to get together as independent citizens in order to achieve certain important objectives." These social and cultural issues are intimately part of environmental management as suggested by the oft-quoted dictum in the Victoria University of Wellington Masters of Environmental Studies programme, "environmental management is $90 \%$ about managing people and $10 \%$ managing the environment."

Having an interest in social and cultural issues does not require seeking solutions to specific problems and instead it is valuable to simply understand what a situation is like, without having to focus on changing it. As such this research is not actionoriented and I am very cautious about making action oriented "this is how it should be..." type statements as, for example promoted by the discipline of human ecology (Weyns, 1998) without first very thoroughly seeking to understand how the situation is. This paper intends to provide some fuel for discussions surrounding what the context of the journey to school is like and how this issue is conceived. 


\section{A Review of the Walking School Bus Phenomenon}

Riding on a wave of popularity WSBs are growing in popularity both nationally and internationally (Kearns and Collins, 2003). For example by March 2000 "Zippy” had been launched at Gladstone Primary School, Auckland (Kearns et al., 2003) and also three smaller WSBs had been established in Christchurch. Although I found a paucity of accessible and up-to-date monitoring of the state of WSBs for Wellington, anecdotally I have found that a similar number of WSBs were established in the capital concurrently. Having been established, the WSB phenomena have continued to multiply and are seen to be a user-friendly way of getting to school (Collins and Kearns, 2001). "Over the last year, the number of walking school buses has increased by 230 per cent, with more than 240 walking school buses now taking around 3,000 children to and from school across the country," says Judith Tizard, Associate Transport Minister (Scoop, 2004). Similar success stories in terms of positive outcomes associated with WSBs exist internationally, for example Bickerstaff and Shaw (2000) evaluated a WSB scheme in Staffordshire, UK and suggest numerous positive effects stem from with WSBs, including an attitude shift towards green travel plans.

WSBs have widely been found to offer a beacon of hope in the face of mounting concerns that surround the journey to school. For example, O’Fallon (2003) describes the attractions of WSBs to include; reducing the number of cars on the road around the school, building a sense of community, and affording caregivers extra time by providing a convenient alternative for the journey to school. Further, children have a safe and non-polluting way of getting to school that is a healthy form of exercise and through which they can develop their own road safety awareness that gives them a sense of independence while being part of a team.

Reports that promote the environmentally friendliness of WSBs have been meet with a certain degree of interest and enthusiasm by many authoritative stakeholders in the journey to school community, including, but not exhaustively Energy Efficiency and 
Conservation Authority, Local Government Authorities, the Land Transport Safety Authority, and the Ministry of Health. There are, however, some voices, such as Baddeley (2000) that allude to the complexity of addressing the issues of automobile use that surround the journey to school. Baddeley (2000) suggests that it is uncertain how much congestion can be eased by reducing the use of automobiles for the journey to school, such as through increasing WSB patronage. Baddeley (2000) likens the automobile congestion to a gas, which fills up all the available space, rather than being like water, which will circulate better in less congested pipes. Using this analogy it is uncertain whether WSBs would ease the overall road congestion issue. Nonetheless Baddeley (2000) provides for at least some hope that WSBs have at least some positive impacts. Traffic congestion is eased in the immediate vicinity of schools and the socialisation of another generation into autodependence is delayed.

WSBs should not be overly romanticised as a mode of travel that is without issue. In addition to Baddeley's (2000) suggestion that WSBs will appease traffic congestion only to a limited extent, it needs to be recognised that neither do WSBs allow for a return to the nostalgic past that caregivers recall of their own carefree school days. While WSBs allow the development of some independence, Kearns et al. (2003) suggest that they can also been seen as a form of social control that does little to encourage empowerment and self-determination in children as they mature. So the horizon of hope provided by WSBs is somewhat clouded and this mode of travel needs not to be idealised.

There is much literature available that focuses on what can be seen as the outcomes of WSBs. For example, numerous papers investigate the environmentally friendly impacts of WSBs and there are other papers that consider how to best establish more WSBs. However, there is a discernable lack of literature looking at WSBs from the opposite direction. In other words focusing on where WSBs come from rather than what they achieve. While both perspectives on WSBs are related, there is a gap in terms of coverage of the former, including consideration of the social and cultural 
ideas that are found to lie behind the different modes of travel used for getting to school.

Of all the literature, Kearns et al. (2003) and Collins and Kearns (2001) are two papers that are of much relevance. As part of their analysis of what WSBs achieve, Kearns et al. (2003) and Collins and Kearns (2001) provide an interesting discussion of what the context is like from which WSBs emerge. A wide range of issues are considered for what lies behind WSBs. The marginal status of walking is recognised and explained as being reinforced as a result of this activity receiving little support in terms of physical infrastructure, such as adequate footpaths. Further walking, as an activity for children is perceived as unsafe as a result of the threat of abduction.

Caregiver concern surrounding walking is described as being further underscored by road safety fears. These safety concerns associated with walking are related to a reduction of social interaction in neighbourhoods. Caregiver fears and the marginalisation of walking are understood in terms of a parenting culture that positions adults as central in their children's lives and encourages a high level of child supervision. So it is within this social and cultural milieu in which Kearns et al. (2003) and Collins and Kearns (2001) position the journey to school.

The discussion of the context of WSBs by Kearns et al. (2003) and Collins and Kearns (2001) provides valuable background in which to locate this paper. However, it is necessary to go beyond what these two papers undertook and to compliment this work with an investigation considering similar issues but from a different perspective. For example, Kearns et al. (2003) focused on what WSBs achieve in the way of benefits. This involved analysing a range of different impacts that were linked to WSBs. Rather than focusing on what positive outcomes can be associated with WSBs, the research interest of this paper is what are the inputs that lie behind WSBs and how do they compare to those inputs behind non-WSB modes of travel. Amongst many positives attributes, such as children developing road safety awareness, Kearns et al. (2003) found that WSBs achieve little in the way of giving children a space in which they have greater independence and the ability to express 
themselves. They conclude that WSBs provide little distinction from motorised modes of travel in terms of young people re-claiming the streets. By investigating indepth, both what lies behind WSBs and non-WSBs, it is possible to provide an explanation for Kearns et al.'s (2003) conclusion.

In the introduction I have already briefly described what attractive impacts WSBs have been found to produce and it is unnecessary to labour on this further. Analysis of the impacts of WSBs have been discussed elsewhere. The focus of this paper differs somewhat from available literature on WSBs and other modes of travel to school and I find little merit in reviewing other studies that, while possibly interesting in their own right, do not contribute to a wider understanding of the social and cultural context of the journey to school.

I will provide O'Fallon, Sullivan, and Hensher's (2002) research as an example of how this paper differs from existing work. Note, that it is neither relevant nor intended that I bring into question the quality of their work. O'Fallon et al. (2002) undertook an experiment investigating the constraints affecting decision-making by morning commuters, which involves amongst other things the journey to school. The method they used involved conceptualising the situation as a black box in which some variables shaping the journey to school are manipulated and the resulting outcomes are recorded. O'Fallon et al. (2002) looked at what lies behind different modes of travel by considering variables individually. A limitation with this view is that it is difficult to see the relationships between commuters' lives and wider environmental systems. I aim to compliment work, such as O'Fallon et al.'s (2002) by casting a little light into the black box. This paper aims to draw together the themes that have been dealt with in other studies so that they can be understood as part of a wider system.

\section{Structure of this Paper}

The research has been briefly introduced in this chapter and it is apparent that the journey to school is a significant contemporary environmental issue, surrounding 
which are a number of important social and cultural issues. Before outlining the concise research focus at the end of Chapter two, I will describe the viewfinder through which this question is addressed. The third chapter begins with an examination of some methodological issues both specifically relating to interviewing as the adopted research technique and to research more generally. Moving on from these issues a more practical overview of how the research was undertaken is presented. Chapter four provides a selection of what was found through a comparison of WSBs and other modes of travel in terms of a range of social and cultural dimensions that were discussed in the interviews. Then, Chapter five considers how the comparisons in Chapter four illustrate the organisation of the journey to school. The paper concludes in Chapter six by reviewing the limitations what this paper did not achieve and then finishes with a summary of what has been achieved. 


\section{Chapter Two}

\section{The Journey to School Environment Through a Social and Cultural Viewfinder}

This thesis focuses on a number of social and cultural dimensions of the environment surrounding the journey to school. Before presenting some more practical aspects of how this research was conducted in Chapter three, this chapter will describe what the social and cultural viewfinder is like for this paper and will finish with a concise statement describing the research focus. The social refers very broadly to how groups of people in the environment relate to one another. Adapting Fertig's (1996) suggestion about how to analyse a situation from an anthropological perspective, I grouped social relationships into three categories and used these as a guide to build up a structural picture. The first group can be labelled economic aspects, and includes things such as the division of labour, the type of occupation, and the division of resources. The second group broadly refers to social organisation. This includes patterns of cooperative behaviour involved in reproducing a culture's membership, such as family structure. The third group covers political activity, including patterns of leadership and power. In addition to considering the environment in terms of its social dimensions, my viewfinder also focuses on the cultural dimensions.

The cultural refers to the meanings that we use to organise and make sense of the environment (Rosman \& Rubel, 1995). For example, the journey to school is indelibly connected to the symbols that we attach to different elements of the environment. The journey to school can be seen as developed through a transfer of symbols both internally and socially (Geertz, 1973). Largely these symbols are developed through words, but can be anything that is removed from reality and that is used to impose meaning on experience, including through drawings, musical sounds, and mechanical devises. I accounted for some cultural dimensions of the 
journey to school by investigating the meanings that caregivers associated with how their children travelled.

Accounting for social and cultural parts of the journey to school imbues it with a wide range of dimensions. I propose, using the social and cultural categories, to broaden what has been conventionally understood as a journey. Conventionally, a journey has been described, as for example by Kokotailo (2000) as involving a number of trips that are defined in a narrow sense. As such a journey could comprise of a bus trip and a trip from the bus stop to school. However, through a social and cultural viewfinder it is possible to broaden the conception of what a trip is. For example, Solnit (2000) suggests that walking can be seen as an expression of love or as a space for thinking. By including the social and cultural aspects of the journey to school it is possible for a trip to traverse more abstract aspects of the environment. For example, the journey to school involves a trip in terms of a child growing up and developing greater independence from the home. Note that I am complimenting my consideration of the social and cultural aspects of the journey to school by investigating some physical dimensions, such as the distance between home and school.

So far I have largely described the social and cultural context of the journey to school as being made up of many different parts, which can be categorised into a number of groups. For example, the journey to school is shaped by economic and political elements. However, to thoroughly understand the journey to school environment I need to compliment my consideration of parts with a thought to how the parts relate to each other (Rosman \& Rubel, 1995). For example, do fears about stranger danger and traffic relate? Are they part of a wider system of organisation? So, as to address questions such as these, consideration is being given to aspects, such as how can individual caregiver ideas be tied to processes in a wider community context and how are these organised. 
The role of choice and determinism in society are relevant to a discussion of how the parts of the journey to school are organised and how this process shapes caregivers' lives. Through interviewing caregivers it became apparent that it was misleading to conceive of the journey to school as shaped by choice. This acknowledgement meant that I had to change the initial research focus, which was to investigate the social and cultural system behind caregivers' choices in the journey to school. While individual agency has some role in shaping what people do, it was misleading to afford this influence too much significance (Giddens, 1987). For example, as one WSB caregiver explained “... it was no decision-making process really... it was just something that you have to do". So I came to see that the web of social and cultural factors behind the journey to school is not entirely shaped by a deliberate rationalisation process. Instead there was also a web of structure that constrains people's lives (Brittan, 1996). For example, on one level children have some input but they are also constrained by what caregivers want, who in turn are constrained by a number of factors.

In considering how the social and cultural parts are organised I view that a balance exists between individuals being able to choose what they do and being tied to something bigger that they cannot choose. Much earnest debate surrounds where the balance lies between structure and agency. However, it seems that the issue is unresolvable in terms of a definitive answer and instead in the face of an ideological impasse it is appealing to reconcile the issue by suggesting that a middle ground exists between the two extremes, as Bourdieu did in Rapport and Overing (2000). For example, I can with some confidence suggest that I can choose the colour of a car that I might want to buy. However, it is less clear whether I have the same choice about whether I want to be part of a highly autodependent society. Structure and agency coexist and interact to such an extent that structure is meaningless without acknowledging agency and vice versa (Brittan, 1996). So through my viewfinder I recognise that the journey to school involves some balance between the simplistic dualism of structure and agency. 
Having introduced the important concepts of the social and the cultural I propose that these are seen as integral parts of the environmental system and are therefore of relevance to a Masters of Environmental Studies thesis. Goudie (2001) claims that there has been a shift in environmental disciplines to integrate them more closely with human affairs. However, this shift has not lead to a widespread embracing of the social and cultural dimensions as central to, and not marginal to the study of the environment (Newby, 1997). For example, White, Mottershead, and Harrison (1992) recognise that human societies are an integral part of the environment. Nonetheless White et al. (1992) seem reluctant to give more prominence to the social and cultural processes as part of the environmental system. This distancing of the social and cultural processes from the environment overlooks the relationships and blurred boundaries between them.

By viewing the social and cultural dimensions of the journey to school I attempt to broaden the understanding of it. Accounting for the social and cultural helps explain how people make sense of what they do and what goes on around them. For example, there are so many things going on around us that we organise things through a process of simplification (Douglas \& Wildavsky, 1982). In this process people can be seen to "act less as individuals and more as social beings who have internalised social pressures and delegate their decision-making to processes of institutions (Douglas \& Wildavsky, 1982:80)”. The arrangements of knowledge underlying what people do for the journey to school are not random. Instead it is possible to see a social and cultural system that produces particular arrangements of knowledge. Viewing these organisational processes behind what people do in the journey to school forms the basis of this thesis. Note that it is not the role of this project to make simplistic judgements about whether the generalisations produced through this organisation process are right or wrong. In other words I am not assessing whether the generalisations that lead to WSBs or other modes of journey to school are good or bad. Nor am I evaluating the impacts of various journeys to school. 
By investigating the journey to school as part of a social and cultural system I am viewing it beyond a set of categorised factors, such as convenience, risk, and rising car ownership (School Transportation Group, 2001). Rather than just seeing risk as shaping the journey to school, it is valuable to consider the more subtle aspects of what risk means and how it interacts with other factors, such as parenting culture. This complexity and interrelatedness of the journey to school (Department of Transport, 2003) is accommodated by viewing the journey to school as part of a social and cultural system. As a system I consider both the individual components, while also seeing how these relate to the whole. This whole is important for, as Fritijof Capra (in Suzuki \& Dressel, 1999:15) eloquently put it, "the whole is greater than the sum of its parts".

The need to understand the journey to school as part of a wider social and cultural system is presented by Black, Collins, and Snell (2001). The journey to school is described as a very complex issue, which needs to be understood by considering the relationships between a wide variety of environmental factors. Black et al. (2001) acknowledge the existence of a large-scale interconnected system that shapes the journey to school, which is beyond the control of the individual. However, they suggest that consideration of such a system is beyond the scope of their study. I aim to address this gap by exploring how a social and cultural system surrounds the journey to school and will in the following section provide a concise description of the research focus.

\section{Research Focus}

This thesis seeks to address the following central question: do WSBs emerge from a different or similar social and cultural environment when compared to other modes of travel used for the journey to school? To this end this paper aims to:

- Explore a range of social and cultural dimensions of the journey to school involving WSBs and other modes of travel; and

- Compare and contrast some key social and cultural dimensions of the background for WSBs and other modes of travel. 


\section{Chapter Three}

\section{Methodology}

\section{Introduction}

Having established what this paper is focusing upon, the attention now turns to a description of how the social and cultural dimensions of the journey to school were studied. Interviewing is the research technique being used for this research and is highly popular (Denzin \& Lincoln, 2000). However, interviewing involves a number of methodological issues. In particular, interviewing is one of many research techniques that focuses on words (Fontana \& Frey, 2000). However, only part of the world is revealed through words though and significant non-verbal dimensions of conversations are overlooked (Poland and Pederson, 1998). After acknowledging the importance of non-verbal dimensions of interactions and that these are largely overlooked through interviewing the limitation surrounding the use of normalised responses is considered. In addition to the limitations of interviewing, recognition is given to the shortfalls that surround the research process more generally, including subjectivity and how this is to be reflected. The rest of this chapter is dedicated to outlining the more practical aspects of how this research was undertaken.

\section{Interviews Involve Normalised Responses}

In addition to the issue of words displacing silences I found that the words themselves presented additional challenges. Due to the difficulties of interpreting silences I acknowledge that I overlooked these important dimensions of conversation. Instead my attention was more readily directed to more tangible words. However, with interviewing what is said maybe as misleading as enlightening in terms of an understanding of the situation. Bradburn (1983 in Fontana \& Frey 2000: 650 ) describe that a major obstacle in interviewing is the "social desirability" phenomenon, where respondents form an impression of the socially acceptable, preferred response and they go on to feed the interviewer with what they think they want to hear. These normative responses "may involve a string of cliché phrases that 
are learnt responses that serve to obscure the realities of their life details more than reveal them." For example, it could be that many caregivers romanticised walking as something that they did often. Walking is something that caregivers see as healthy and they like the idea that they do this often and present this image. Another example of difficulties involving normalised responses could have surrounded how some caregivers talked about the importance of their children developing healthy habits, while other caregivers did not make this explicit. Just because a caregiver presents the appearance that it is important for their child to develop healthy habits, does not necessarily mean that their child will actually be given greater encouragement compared to a situation where caregivers did not make this explicit.

\section{Reflecting on the Limitations of Interviewing}

While not really overcoming interviewing's limited ability to account for non-verbal dimensions of a situation, it is still valuable to acknowledge this shortfall and reflect on what lies behind this trend. The popularity of interviewing needs to be understood within a wider societal context, in which talk and action are valued over listening and reflection (Poland \& Pederson, 1998). For example, a skim through the classic texts (Fielding, 2003) on interviewing, such as Spradley (1979) reveals that talking is the

key principle of success and silence represents failure. Further, Poland and Pederson (1998) suggest that neglect of listening is likely to stem from the reason that those who control the production of knowledge are more concerned with airing their own views than with hearing others'. This brings me to the important acknowledgement that, to a certain extend at least, this project reflects what I want to hear and say.

\section{What does Research Mean Anyway?}

The acknowledgement of these limitations confronts a number of more general methodological issues surrounding most, if not all research. These include, what do my observations mean? What is my place in the research and how do I reflect this? And even what dangers surround trying to talk your way through these? Central to these questions is the recognition that interpretation of the journey to school involves research, which Bourdieu (2003) suggests is a process that is limited and subjective. 


\section{Recognising the Subjectivity of Research}

The story that I tell through this thesis has to be recognised as subjective but also as something that is enriched by seeing it from outside of the lives of those involved. Through a narrative process (Silverman, 2000) I have built up a picture of the journey to school through a two-way arrangement of giving and taking information with the informants (Fontana \& Frey, 2000). The resulting understanding needs to be grounded in the context of the interview (Gibbons, 1987). For example, what I saw and how I configured these findings is intimately subjective, so that what I observed would be different to another person's observations in different circumstances. For example, this research involved qualitatively comparing and contrasting caregivers' comments about a range of different social and cultural dimensions of the journey to school. Judging whether and how much there are similarities and differences can be highly subjective. When, for example are similarities judged meaningful and when are they not? At one level it is easy for a caregiver to say something vague, like exercise is important for their child and for another caregiver to say something sounding very similar. However, these two comments could involve quite different understandings.

So how can this be systematically addressed? To begin with, let it be clear that I am not making claims about having authority as a researcher as discussed by Sangren (1992). Instead I have simply tried to bolster credibility in a couple of ways. Firstly, during the interviews I sought to clarify with caregivers what they meant in relation to other comments by teasing out how they responded and acted in relation to what they said. This relates to the importance of asking how rather than why in interviews as picked up during the coding of interviews and suggested by Tolich and Davidson (1999). Secondly, I attempted to make the process more robust by being as accountable as possible. As suggested by Lofland and Lofland (1995) I have tried to give the reader as much detail as possible to reinforce the argument. 


\section{Limits to Subjectivity}

The recognition that interview observations are the product of a subjective process need not to be taken too far. I have not adopted the idea of the Romantic Movement, that "the nearer we come to the respondent, the closer we are to appreciating the "real self" (Fontana \& Frey, 2000: 664). It is misleading to try and understand a situation by trying to get entirely into someone else's shoes (Dingwall, 1997). For example, I cannot make sense of everything that someone else does by trying to see the situation through their eyes. Marx, Freud, and Nietzsche (Gibbons, 1987) see that there are limits to self-understanding and as such I recognise that the environment is not entirely subjective.

\section{The Importance of Reflecting About Research}

The adoption of a reflective approach to interviewing provides a satisfying way of interpreting the interviews. Reflectiveness is an iterative process through which I work to illuminate interviewees' worlds using my own understanding (Ellen, 1984). Through this backwards and forwards process, I have not objectively tried to measure how people construct and are constructed by their environment. Instead Lofland and Lofland's (1995) suggestion has been adopted so that by openly having one foot in my world while trying to get the other foot in their world the collection of a rich data set is assisted.

\section{Avoiding too Much Reflection}

I will limit this discussion of the methodological issues surrounding this thesis with a word of caution regarding the excesses of self-reflectivity in much recent scholarship (Lofland \& Lofland, 1995:14). It seems ironic that while acknowledging that research is a subjective affair, numerous authors seem to attempt this through prolonged self-reflectivity. So as not to fall into the trap of trying to reduce the complexity of my position I believe that it is expedient to limit the attention given to this task. Instead I recognise that limitations exist at both ends of the scale, from conventional attempts to achieve so-called objectivity and distance in relation to the field to the excesses of self-reflectivity (Ellen, 1984). 


\section{On the Practice of Semi-Structured Interviewing}

Following the discussion of some theoretical issues surrounding research it is appropriate to present the more practical aspects of what I did. I used a semistructured interview style, as I wanted to be able to repeatedly cover a wide range of issues in a way that could be suitably adapted to fit the different interview situations. Being semi-structured I could tailor the interview style and was not constrained to a set format (Fielding, 2003). I was attracted to Bernard's (2002) suggestion that semistructured interviewing is the most appropriate interview style in situations where the researcher does not have large amounts of time for repeated meetings with informants.

\section{Interviewing with an Aide Memoir}

The interviews used in this research where greatly assisted by an aide memoir, as attached in Appendix 1. Although this guide can appear to be a very structured set of questions for a semi-structured interview, it was not applied in such a way so that the flow of talk was heavily structured. Instead I used the aide memoir as a lose checklist that certain issues had been dealt with, in an order that was not controlled. The aide memoir helped ensure the balance of getting people on topic while getting out of the way to let the informant provide the information that he/she thinks is important (Bernard, 2002).

\section{Convenience Sampling}

Because of the research focus for this project it was not necessary to select a specific or representative population sample. As suggested by Tolich and Davidson (1999) I did not require fitting the sample to the research question. I was not investigating how the journey to school was organised for solo-mothers and did not need to work with caregivers from selective groups, such as solo-mothers. Niether am I claiming to represent what the context is like for the journey to school generally. Instead the focus of this research is to investigate how the mode of travel used in the journey to school relates to the social and cultural context. As described in the research focus I am considering a range of social, cultural, and physical dimensions of the journey to 
school. An understanding of the relationship between mode of travel and social and cultural background can be developed in a range of situations. Controlling the specific context is not important when looking at how social and cultural processes shape the journey to school. As this was the research interest it was not necessary to use specific selection criteria and as described by Bernard (2002) I could use convenience sampling for this exploratory research.

\section{Interviewing two Groups of Caregivers}

Interviewees came from two broad groupings: caregivers whose children use a WSB and all caregivers whose children use some other mode of travel for the journey to school. I acknowledge that the grouping of journeys to school into two categories, WSBs and non-WSB, is very rough. For example, by lumping together all non-WSB journeys to school into one category it is unsurprising that I found similarities between at least someone from within this broad group and someone from the WSB group. This potential crudeness seemed to be the product of an inevitable trade off, as I wanted to explore WSBs by comparing and contrasting them with other modes of travel used for getting to school.

Caregivers were interviewed, as they are a dominant factor in determining the mode of travel used for the journey to school (Kearns, et al. 2003). For each household I interviewed one caregiver, and I did not seek to talk with all caregivers of the children. Although caregivers sometimes talked about their partner this data set was very incomplete and has not been brought into the analysis. Although it would have been interesting working with children to investigate their perceptions of the journey to school, I declined to do so for a number of reasons. These include being deterred due to fears of being persecuted from working with young children, especially as I am a male. In this climate it is easier to simply justify working with caregivers and to focus on how they see the journey to school. WSBs have been targeted at primary school age children and this dictated that I was to talk with parents from this group. 


\section{Interviewing ten Caregivers}

Although the decision to work with ten interviews involved a change of plan from the initial eight, I think that this was sensible due to a number of factors. Firstly, it was difficult to know at the time of the interview whether it was going to be valuable or not. So I figured it would serve as insurance to have a couple of extra interviews up my sleeve while allowing me to be able to bundle all my fieldwork together. Ten interviews of approximately one-hour in duration were selected as the research focus required depth and not breadth. Using a budget of six to seven hours of transcribing per one-hour interview, ten interviews fitted the constraints of this thesis.

\section{Sampling from two Schools - Variation and Bias}

By dividing my convenience sampling between WSBs and non-WSBs and different decile schools it seemed plausible that this would provide a window on what lies behind the journey to school in a range of situations. By arranging caregivers into WSB and non-WSB groups I was able to compare and contrast various social and cultural dimensions behind these modes of travel. This meant I was able to build up a picture of how the context of the journey to school was organised and what was going on as part of a social and cultural system. Note that the specific size of the decile gap between the two schools was not significant. So I scoped the feasibility of nine schools within the Wellington area that were involved with WSBs and settled with one lower decile school and one from a higher decile community. I decided to only work with schools that had WSBs. This was done in the aid of feasibility because I would have access to both WSB and non-WSB caregivers within the same school community. Note that both of the schools that were selected were of relatively close proximity to the city centre. It is likely that by both being in a central location this creates a certain bias in my sampling. For example, it is likely that the communities that I investigated were busier in terms of people walking to work, due to the proximity to the $\mathrm{CBD}$, than would be found in schools with more of a suburban setting. This reinforces the limitation that this study is not representative of the wider population. 


\section{Selecting Caregivers}

Initially I thought I would select my informants from within a walking radius of the school defined by the outer most extent of WSBs for that school. However, this category was eroded by a number of factors. These included that nine of the ten interviews were with mothers and for this reason I was interested to include the one father who registered an interest in participating in this study. However, he lived almost five times the distance of the outer most extent of the WSB for that school. This was one of the reasons why I discarded trying to use walking radius as a selection criterion. Further, it became apparent that a clear delineation between categories of walkability and non-walkability did not exist. I came to realise that caregivers' assessment of walkability was part of what I sought to uncover through my research and this was not something that could be neatly determined from the outset.

Having selected two schools, contact was established with caregivers through the schools' newsletters. I hoped that at least two caregivers from each category in both schools would register an interest and then be willing to participate. However, the response rate was only tepid. One caregiver directly contacted me while five others returned the slips posted in the newsletter to the school office. Consequently there was a shortage of caregivers, especially of those who were involved with WSBs. Thankfully the principal of each school provided me with an additional informant. With these contacts I was then able to snowball other informants to fill my quota. Only one parent who volunteered to be involved was dismissed on the grounds that I already had sufficient numbers of participants with non-WSB affiliation. So in the end, four of the ten interviews were with WSB caregivers and the remaining six were with non-WSB caregivers.

\section{Conducting and Taping the Interviews}

All interviews occurred between July and September 2003 and took place within a 95 Monday to Friday timeframe. The interviews took place in a range of situations, including caregivers' homes, their offices, their classrooms, and their school staff 
rooms. Interviews with caregivers were one-on-one. The length of interviews ranged from about half-an-hour to about an hour and all the interviews were tape-recorded. This was planned, as it was unrealistic to commit the interviews to memory (Silverman, 2000). Further, frantically scribbling notes in an attempt to capture some immediately obvious important points is also likely to be stilting (Tolich \& Davidson, 1999). A recording was also useful to work with for subsequent analysis. Although recording the interviews had the potential to hobble the flow of conversation (Fetterman, 1989), I accepted this risk. In hindsight it seems that the presence of the tape recorder had no tangible effect. For example, the interviews had a similar flow both on and off tape. I think this was possibly due to establishing strong rapport with participants and having an at-ease atmosphere.

\section{Coding}

Transcribing the recordings provided an opportunity to start the coding process as soon as I had undertaken the first interview. As is commonly recommended to the uninitiated student, for example Tolich and Davidson (1999), Hutchby and Wooffit (1998), Lofland and Lofland (1995), I transcribed the recordings myself. I transcribed the recordings by playing the tapes backwards and forwards while typing up the conversations. Transcripts varied in length from seven to fifteen pages.

Through this process I was able to reflect on my work and identify what worked and what did not.

\section{Feedback from Coding on the Interview Technique}

Based on reflections of earlier interviews I was able to address some weaknesses that were of concern and might undermine the quality of the data set. The weaknesses included, a heavy reliance on the prompt why. After undertaking my first interview I became aware that it was common practice for experienced researchers to avoid asking why (Tolich \& Davidson, 1999). Instead it was recommended that it was more constructive trying to capture process rather than outcomes. I came to better appreciate that it is the role of the researcher to gather the richest possible details about the process and to subsequently theories about them (Lofland \& Lofland, 1995). By relying heavily on "why" I was potentially intrusive and invited 
unprepared and misleading response. So I adapted my focus to look at how things were done.

Effective probing is essential to successful interviewing (Bernard, 2002) and through the coding process it became apparent that I had some issues surrounding the questions and responses used in the interviews. I found it very hard not to ask leading questions and give biased responses. In my eagerness to make the interviews as non-threatening and casual as possible I often found myself giving such enthusiastic responses full of encouragement that they were also heavily biased. It became apparent that a tension existed between the widespread discouragement of interviewer bias and the idea that interviews are based on the establishment of shared meaning (Fontana \& Frey, 2000) and I struggled with the ambiguous distinction between these. For example, how much could I support caregivers and how much did I have to distance myself. A similar issue surrounding my response to caregivers was that I struggled to distinguish between asking open questions and asking vague and ambiguous questions. I attempted to manage these issues by being as non-leading as possible and by then giving reflection to this issue here in the thesis.

Through the coding process it was apparent that the research focus had narrowed to the journey to school and not home again. The majority of interviews were spent talking with caregivers about the arrangement on the way to school. Consequently the data set was too sparse and incomplete in regards to what lies behind the homewards journey and the research question was adjusted as appropriate. As such, it is the environmental context of the journey to school that is investigated and while this may well be similar in many respects to the journey home again, it is not appropriate to discuss this relatively unexplored territory. Instead more research would be needed to discuss the journey home again.

Another aspect of the initial research focus, which changed, was that I had set out to look at what lay behind the emergence of WSBs. However, the scope of the discussions that I had during the interviews was too limited to comment on what lay 
behind a process of social change. As they stand, the interviews do not contribute to a significant understanding of the historical context. To suggest what lies behind the emergence of WSBs it would require a greater focus on how the environment has changed. The interviews in this project do not introduce any great depth of time. As the scope of this project was limited the research question was adjusted to focus on the contemporary context of the journey to school.

\section{Conversation Analysis}

As I relied heavily on talk to inform my research it was necessary to consider how I was going to negotiate my way through analysing the interview conversations and what I would bring into my research from the field of conversation analysis (CA). Having briefly surveyed discussions surrounding CA it was apparent that this paradigm (ten Have, 1999) requires analysis involving a high level of detail. Transcription for CA is often formidably complicated to the untrained eye (Hutchby \& Wooffit, 1998). Although it is possible to overcome this lack of fluency, this is unnecessary (Lofland \& Lofland, 1995) for the scope of this project. A 100\% systematic approach was, and remains impossible. I did not need to adopt the methodological approach of CA to analyse the social interactions that inform my research. I was not interested in trying to discover meanings behind social situations through very in-depth attention to detail (Psathas, 1995).

\section{Identifying Common Themes}

Coding transcripts was a central part of building up this project through a process of organisation and synthesis (Flick, 2002). In the coding process the central research interest was related to data that came out of the interviews. A first step in the coding process involved looking through the interviews and identifying a number of common themes. The aide memoir covered an overwhelming range of different aspects of the journey to school. By weaving some of the common threads together I ended up with a limited and therefore more manageable number of categories, such as Comments Reflecting Caregiver Ideas About Environmental and Exercise Value for Children as presented in Appendix 2. These categories were built up by repeatedly reading through the transcripts and listening to the recordings. While 
these can appear as isolated categories from one another they need to be recognised as overlapping. Through a combination of transcripts, recordings, and codings I built up a multi-layered picture of the journey to school that can only be partially presented in text. The coded categories built up from the interviews provided a basis for a comparison of WSBs with other forms of tranport. From these comparisons a number of key themes shaping the journey to school were identified and it was apparent how the journey to school related to caregivers' wider lives and society.

\section{Summary of Methods}

In summary, in-depth interviews were used to investigate how a range of social and cultural factors shapes the journey to school for ten caregivers. These social and cultural considerations involve describing the relationships between caregivers and the journey to school environment and the meanings that caregivers imbue this environment with. As well as a description of the social and cultural parts, for example caregiver attitudes towards walking and ideas about risk, it is considered how these parts are organised as part of a wider context. Using interviews to investigate these phenomena develops only part of the picture and some dimensions, such as the non-verbal ones, are neglected. As such, it is appropriate to recognise that the findings as presented in the next chapter are inherently biased and contain limitations. 


\section{Chapter Four}

\section{The Findings are put Forth}

\section{Introduction}

WSBs shared similarities with other non-WSB modes of travel in a number of different social and cultural categories. Little support is found for the idea that WSB caregivers come from some entirely distinct background compared to caregivers who use other modes of travel. WSB caregivers were found to share similar ideas about the value of an environmentally healthy, safe, cheap, and convenient journey to school with non-WSB caregivers. For example, both groups of caregivers recognised the benefits of their child getting fresh air and exercise in the journey to school. As suggested by proponents of WSBs, such as O'Fallon (2003), some variation was found between different journeys to school, such as only non-WSB caregivers commenting that they avoided walking. In Chapter five I will consider what these similarities and differences suggest about patterns of organisation behind these findings.

The findings presented in this section are only a selection from the coded data. See Appendix 2 for a more detailed selection of the coded data that has been grouped into categories for comparison. Note that to ensure anonymity of caregivers I have not disclosed gender and other personal characteristics with which caregivers may be able to be identified. The more raw coded findings have been presented in the appendix because they are too extensive to fit coherently in a findings section. Being qualitative research it is not possible to neatly summarise pages and pages of data into graphs or tables. Further much of the data that was collected is not relevant to the research focus of this paper. During interviews it was not possible to direct caregivers to cover exactly what was being focused on. The aide memoir helped keep on task but there were always interesting tangents. Although potentially interesting, many tit-bits have not been expanded. Sometimes one caregiver would talk about something that others did not cover so I was unable to compare such 
information. So an Occam's razor has combed the pile of tapes and hundreds of pages of transcripts and what has been presented is a selection of the coded data that is relevant to my research focus.

\section{Caregiver Ideas About Their Children Being Outdoors}

The value of children being outdoors in the physical environment was something that both WSB and non-WSB caregivers associated with the journey to school. Similar to Kearns et al. (2003) I found that caregivers currently involved with WSBs drew attention to the value of their children being able to be outdoors on their own two feet. The value of this experience was described in terms of the opportunity that it provided for children to ground themselves in their environment. For example, one caregiver described the value of "stopping and looking at things along the way... finding dead insects and... you know looking at trees... and noticing the flowers coming out and stuff." While Kearns et al.'s (2003) research into WSBs did not investigate how non-WSB caregivers valued the environment, I found that both groups of caregivers expressed similar views. As such non-WSB caregivers also acknowledged the value of their children experiencing the environment in a hands-on way. Alternatively a small minority of caregivers, from both groups made no direct reference to the value of just being in the outdoors environment. So this suggests that an explicit appreciation of the environment is not peculiar to WSBs nor does having had involvement with a WSB necessitate a direct reference to the environmental value of the journey to school.

\section{Caregivers on the Stranger Danger Issue}

Stranger danger refers to the risk posed by strangers to children in the journey to school and was a key issue identified by both WSB and non-WSB caregivers. One caregiver involved with a WSB provides an example of this aspect of the journey to school, "there are lots of crazy people rushing around doing things that you cannot trust". Another caregiver involved with a non-WSB journey to school described her perception that "every school has got like a lurker... or something." So similar ideas about stranger danger were found to pervade the different journeys to school. 


\section{Supervision and Community Building in Response to Stranger Danger}

In response to the widespread perception of stranger danger many caregivers from both WSB and non-WSB groupings felt that their children needed to be supervised and that strengthening community cohesion or community building was something that valuably assisted this supervision. Similar to Kearns et al. (2003) I found that caregivers responded to the threat of stranger danger by increasing the supervision of their children. I also found that caregivers recognised the value of community building in terms of supervision for their children for the journey to school. For example, one WSB caregiver described how it made good sense to strengthen community ties to help supervision for the journey to school as "we are after all social beings." Additionally non-WSB caregivers described how they saw the neighbourhood as assisting their child's supervision.

\section{An Opportunity for Children to Develop Healthy Habits}

Many caregivers from both WSB and non-WSB groups were aware that the journey to school provided a valuable opportunity for their children to develop healthy habits. Similar to Kearns et al. (2003) I found that all caregivers involved with WSBs described this mode of getting to school as providing an important opportunity for their children to develop healthy lifestyle habits. For example, a WSB caregiver commented "the journey to school provides a valuable opportunity for children to get some fresh air and exercise before setting into class". Similarly I found that caregivers involved with non-WSBs, including one who used a car for the journey to school, also recognised that walking to school "is an important experience for them [the children] and helps them get psyched up for the day at school". I found that it is not a simple matter of caregivers being able to translate their wish for their children to be able to get some fresh air and exercise into actually obtaining this. Other considerations, such as work commitments were influential. 


\section{Caregiver Ideas About Their Children Developing Independence}

The journey to school was often seen both by WSB and non-WSB caregivers to provide an opportunity for their children to develop some independence. Most of the WSB caregivers discussed the need for their children to develop some independence. For example, one caregiver believes that the journey to school "....provides an opportunity for children to develop the healthy habit of walking to get places rather than becoming reliant of sitting in a car to get places". Similarly some caregivers involved in non-WSB journeys to school also referred to the importance of the journey to school for developing independence in their children. For example, one caregiver mentioned “... you know he's old enough to start learning some independence... so I need to start giving him opportunities to start developing those skill[s]". Also the benefits of developing more independence during the journey to school for children is described by another non-WSB caregiver, “... they're more independent when you let them walk... you know they'll be able to use their initiative... I'd say it is good for them because they don't have to rely on me all the time... they don't have to look up to mum to take them wherever".

\section{Shared Responsibility}

Hand in hand with the recognition of opportunity for children to develop some independence, many caregivers from both groups saw that responsibility for the journey to school extended beyond themselves. For example, one WSB caregiver described how, "children in the journey to school also have responsibilities and it is not just with parents." Similarly non-WSB caregivers see that the responsibility for the journey to school is spread beyond themselves. For example, one non-WSB caregiver described how “....it's also the children's and communities responsibility as well... even though the community might not know them [the children] they can still help." Responsibility for children in the journey to school was found to go beyond just caregivers for both WSBs and non-WSBs. 


\section{The Importance of Road Safety}

Caregivers often identified traffic conditions and safety as a significant part of the journey to school. Both groups of caregivers described the journey to school as containing busy traffic conditions and that this was something that required supervision. Similar to Kearns et al. (2003) a key concern for caregivers involved with WSBs was ensuring their child's road safety. Non-WSB caregivers also expressed similar road safety concerns. As well as associating traffic risks with the roads caregivers from both groups described the dangers surrounding cars parked on footpaths and dangerous entranceways.

\section{The Availability of Alternative Modes of Travel}

The availability of alternative modes of travel that could be used for the journey to school was not significant in terms of what mode was actually used. The majority of households, regardless of whether they were involved with WSBs or other modes of travel, had cars available for the journey to school. Although a car was available, caregivers still differed in terms of the modes of travel they used for the journey to school. Further many households, both with WSB and non-WSB involvement, had bikes available. However, as suggested in Kearns et al. (2003) biking is considered unsafe by many caregivers and I found bikes were not used in any of the journeys to school. So it seems that availability alone of various modes of transport does not clearly determine what is used.

\section{Appreciation of Time Spent With Children}

Appreciating the time spent with their children was something that both groups of caregivers recognised as part of the journey to school. Similar to O'Fallon (2001) I found that WSB caregivers commented that they appreciated the time that they were able to spend with their children in the journey to school. Note though that a few WSB caregivers recognised that they missed the earlier journeys to school where they spent one-on-one time with their children. For example, one WSB caregiver commented, "I miss time travelling to school just with my son... it was a nice period for us just to be together... to talk about the day." I found that many of the non-WSB 
caregivers also commented about how they appreciated the time that they spent with their children during the journey to school. O'Fallon (2001) suggests that this is why many caregivers do not use WSBs as they prefer to take their children themselves. It was apparent that caregivers appreciated the time spent with their children and that the specific situation in which this occurred was not significant. A number of caregivers who used the car for the journey to school commented how the time spent in the car could be used as quality time with their children. For example, one caregiver commented "we get to catch up... him and I get to catch up because I don't get to see him once I go to work... I'm not here at nights... so I get to do my parent thing and ask him about his homework and that sort of thing". So being able to appreciate time spent with their children seems to be something that is not restricted to particular modes of travel for the journey to school.

\section{Role Models Provided by Caregivers}

Being a positive role model for their children in terms of using walking, as a mode of travel was something that both WSB and non-WSB caregivers provided. Caregivers from both groups showed positive attitudes towards walking for themselves and for their family. Such enthusiasm translated into many caregivers from both groups walking to work and as much as possible on other general trips. Nonetheless caregivers often enthused about wanting to walk more often than what they did. Caregivers from both groups used walking as a form of recreation. A range of opinions were aired by caregivers from both groups in terms of the benefits of walking, these included exercise, environmental efficiency, and that it was simply a hassle-free way of getting around. One distinction that I found between WSBs and non-WSB caregivers was that comments about avoiding walking only came from two caregivers, both of who were of non-WSBs grouping. These comments were that "I don't walk unless I can help it" and from someone else, "I don't walk even if it's close... yeah I drive everywhere."

\section{How did Caregivers get to School When They Were Children?}

Walking to school during their own schooling days was something that many WSB and non-WSB caregivers did. It is not clear what influence the mode of travel 
caregivers used has on the mode of travel their children use. For example, one nonWSB caregiver described how “...just looking back at when my husband and I grew up... we walked longer distances than they [her children] do now and I thought if I did it and my husband did it then why can't they do it?" Alternatively another nonWSB caregiver remembers, "I always used to see other kids being picked up by their parents... you know my parents never used to pick me up... so yeah I always like the idea of being able to drive my kids to school..." Further a WSB caregiver did not walk to school during her childhood. So although caregivers use a range of different journeys to school now, when they were children it is quite possible for them to have shared the same way of getting to school.

\section{The Impact of Weather Conditions}

The mode of travel used for the journey to school was largely independent of what the weather was like for caregivers from both WSB and non-WSB groupings. Building on Kearns et al.'s (2003) description that WSBs usually run regardless of the weather, this paper found that for two of the three WSBs the weather had to be very wet and cold for caregivers to use an alternative way of getting to school. It was also found that other caregivers who use walking continued to do so unless the weather was very fierce. In such circumstances many caregivers would use a car instead and one caregiver allowed her children to catch the bus. One caregiver uses the WSB regardless of what the weather is like. However, another caregiver using the WSB does not organise the WSB at all during winter due to the colder weather. For caregivers who regularly use a car, the journey to school is relatively unaffected by weather. However, one caregiver who uses a car usually lets her children walk the last section if it is fine. If it is bad weather though the children are dropped right at the gate.

\section{Personal Characteristics}

A number of aspects of the journey to school, some of which are not shown in Appendix 2 for privacy reasons, can be grouped together as they allow no clear distinction to be made between WSBs and other modes of travel. For example, no relationship was found between professional workload, household income, duration 
of the journey to school, and caregiver age or gender. In these comparisons caregivers from both WSB and non-WSB groupings presented similar circumstances. In other words, both modes of travel used for the journey to school come from backgrounds with a mixture of part-time and full-time work commitments with high and low incomes. Furthermore caregivers from both groups presented a range of ages and were able to spend a similar amount of time involved with the journey to school in the morning.

\section{How far is too far - a Comparison of Distance to School}

Most journeys to school were less than four kilometres for both WSB and non-WSB modes of travel. Note that information pertaining to the distance between home and school is not disclosed in Appendix 2 due to maintaining the anonymity of caregivers. The one journey to school that was much greater than four kilometres involved using a car. It seems possible to suggest that for the minority of journeys to school, travel more than four kilometres is unlikely to involve walking because this would be deemed to take too long and would be too much of an effort for children. However, for the majority of journeys to school, which were below four kilometres, it seems there is little relationship between distance and mode of travel. The finding that most journeys to school occur within a four-kilometre boundary is likely to be a generous margin as Black et al. (2001) describe that the majority of children in England live within two kilometres of school.

\section{A Deliberate Decision and the Mode of Travel Used}

Identifying that a deliberate decision lay beneath the mode of travel used for the journey to school was something that caregivers from both groups recognised. Caregivers who acknowledged that such a decision had been made describe themselves as the dominant authority in making the decision. For example, one WSB caregiver described the situation, as "this is what we're going to do!" Alternatively a non-WSB caregiver said, "I decided... I talked about it with my son and suggested that idea and he was keen on it... but I think if he'd said no... it would have still happened... I would have talked him into it." 
While some caregivers from both groups recognised a decision, there were also caregivers from both groups who did not associate a clear decision-making process with the journey to school. Instead the journey to school was something that "just happened," or that "we never really considered any other method of getting to school." So it is not possible to distinguish different modes of journey to school on the basis of one group having more decision-making choice about what they used. Note that while caregivers identified a decision surrounded the journey to school to varying degrees, it was not possible to compare how such a decision was made.

\section{How Much Working Together is There?}

Interacting with other people in the journey to school was uncommon for both groups of caregivers. The organisation of most journeys to school was something that was dealt with inside of one household. In the majority of situations non-WSB journeys to school involved just the one household and often just one caregiver. However, one non-WSB household was involved with a cooperative arrangement with their neighbours, whereby the caregivers from each household took turns getting the children from both households to school. Note that although the caregiver involved in this arrangement described that the sharing occurred "quite often," it was unclear from the interviews exactly how often this occurred. Similar to the common patterns of isolation for non-WSB modes of travel two of the three WSBs were found to rely on just one caregiver each. For the third WSB three different caregivers from two different households were involved on different days of the week. Note that most caregivers had people they could fall back on in out-of-the-normal situations.

\section{Conclusion}

Based on the comparison of a number of different aspects of caregivers' lives surrounding the journey to school it has been found that both WSB and non-WSB groups share many similarities. Caregivers have common ground in terms of their ideas about what the journey to school environment is like. It is commonly recognised by caregivers that it is valuable for their children to exercise and ground themselves in the journey to school environment. However, their children need to be 
supervised in the journey to school due to the perception of two major risks: stranger danger and traffic safety. For most caregivers they were aware that they appreciated the time spent with their children and that walking was something they encouraged. Other similarities also linked WSB and non-WSB caregivers, such as there was often an individual caregiver involved with the supervision of the journey to school. As well as finding similarities between WSB and non-WSB categories note that for most comparisons some variation was also found. For example, some WSB caregivers identified a deliberate decision while some non-WSB caregivers did not. However, this variation needs to linked to the variation that was found within each category. For example, some caregivers involved with WSBs identified a deliberate decision, while others did not. 


\section{Chapter Five}

\section{Analysis}

\section{Introduction - Four Over-Arching Themes}

From the findings it is apparent how other aspects of caregivers' lives shape the journey to school. A number of influences can be found to lie behind the multiple similarities that I found through the comparison of WSB and non-WSB journeys to school. Parenting culture, work commitments, risk, and social fragmentation are four themes that I identified as over-arching caregivers' lives regardless of the mode of travel used for the journey to school. For example, the similarities in caregiver ideas about the importance of fresh air and exercise in the journey to school can be linked to widespread ideas in society that shape what caregivers see as important for their children. The four common themes that are identified link the different modes of journey to school and provide that there is little clear distinction in many social and cultural aspects of their context.

Some differences were found between WSBs and non-WSBs. However, these differences are linked to the variation found within each category. It needs to be recognised that members of each group were not homogenous. For example, some WSB caregivers recognised a deliberate decision surrounds the journey to school while other WSB caregivers did not associate a clear decision. Alternatively some non-WSB caregivers were pro-active walkers, while others from this group were much less enthusiastic towards walking. Following from this variation within categories it is to be expected that variation also is found within the larger grouping of caregivers involved with different modes of travel. This accounts for the variation that was found between WSB and non-WSB groups. Note though that the variation found does not provide for a clear distinction to be established between these two modes of travel. It was found that the many similar ideas that over-arch the different modes of travel are translated in a non-uniform way that provides some sort of guidance for caregivers in the specific mode of travel they use. 
Only four over-arching themes, ideas about caregiver roles, work commitments, risk, and social fragmentation have been identified. However, it is certainly the case that what lies behind the different journeys to school is more complicated than four themes. Nonetheless based on this research these influences were the most significant. Focusing on this number of themes fitted the constraints of this project. I have not, for example, been able to explore the role of gender in the different modes of travel, as no noticeable distinction was apparent based on the interview with the one male caregiver. While I focus on just four themes, I suggest that it is likely that there are additional over-arching influences and that these further enrich the social and cultural system that can be seen to shape the journey to school.

\section{The Pervasiveness of Parenting Culture}

An over-arching system of parenting culture relates to the journey to school. The many similar ideas held by both WSB and non-WSB caregivers in terms of the value of the journey to school environment for fresh air and exercise can be linked to a common system of ideas about what good parenting involves. There is a popular set of beliefs that promotes the goodness of being outdoors, getting some fresh air, and exercise. These ideas are presented to caregivers through many channels, such as numerous and sophisticated parenting advertisements (Beck-Gernsheim, 1996). In this way commonly held beliefs about the value of the journey to school environment for children are found to exist throughout WSB and non-WSB caregivers alike.

The lack of distinction in caregivers' ideas about independence and responsibility can also be related to pervasive ideas that emerge out of parenting culture.

Caregivers live in a society where a huge amount of pressure is placed on them to pro-actively promote their child's development (Beck-Gernsheim, 1996). The encouragement to stimulate their children's development results in caregivers controlling what their children can and cannot do. This control comes in the form of the journey to school being heavily supervised with caregivers guarding their children. So regardless of the mode of travel used for the journey to school I found 
that children had little independence. Instead as found by Kearns et al. (2003) it was common for caregivers to simply take responsibility for providing what they feel is important in the journey to school.

Ideas about caregiver roles can also be related to how decisions about the journey to school were made. The decision about the journey to school was often from the caregivers' perspective. As suggested by Beck-Gernsheim (1996) the decision usually suits caregivers' lives and is made from their position. Little attempt is made to account for the ideas that the child might have to offer and to make them more in charge of what happens. It was common for caregivers to think about their children's needs, such as protection from stranger danger. However, as suggested in Kearns et al. (2003) the response to this need is such that it primarily suits caregivers. I will not discuss whether this fitting in with caregivers is inevitable. Nonetheless children had little input into the journey to school and decisions were often made so that caregivers got what they wanted. In some situations this occurred in the pretence that the journey to school was the child's decision. For example, some caregivers joked how they would have twisted their child's arm so that the caregiver got what they wanted.

\section{The Role of Work in Caregivers' Lives}

In addition to widespread ideas of parenting culture a pervasive work culture also plays a key role in shaping the journey to school. Through caregivers' comments it became apparent that a primary constraint on the journey to school was what work permitted. For example, one caregiver described how she used the car because they "have to dash across town after dropping the kids off at school to get to work on time." The priority given to work by caregivers is symptomatic of a wider trend that society is dominated by work (Swift, 2002). Such a trend can be seen through many New Zealanders putting increasingly long hours into their work (Department of Labour, 2003). As work commitments have an important influence in many caregivers' lives it was common to find that this was a key factor shaping the journey to school. The different modes of travel used for getting to school are all shaped in 
similar ways by the importance given to work commitments by caregivers. Work commitments commonly exist for caregivers immediately after they have taken their children to school. What happens therefore, in the journey to school is closely determined by whether it fits with the caregivers' subsequent work commitments.

\section{Fear is Widespread in a Risk Society}

As well as work commitments some over-arching ideas about the risks involved with the journey to school can be seen to lie behind the different modes of travel alike. Why, for example, did I find that both groups of caregivers perceived the risks associated with stranger danger and road safety more acutely than the health risks surrounding a sedentary lifestyle? Ideas about risk are socially and culturally constructed (Douglas \& Wildavsky, 1982), and are so pervasive and influential that western society has been coined a risk society (Beck, 1992). Both WSB and nonWSB caregivers are bathed in similar ideas about risks. This can be related to the similarities found in terms of stranger danger and traffic risks being identified by both WSB and non-WSB caregivers.

\section{Patterns of Social Fragmentation}

The journey to school is shaped by the individualist lifestyles of many caregivers. As described by Landry (1995) cities are becoming increasingly socially fragmented. Caregivers have to negotiate the journey to school through an environment that involves a community that is not defined in geographic terms. Instead communities are being reconfigured and are defined on the basis of common interests. At the neighbourhood level there is often no sense of community, as the factors that give rise to it, such as social homogeneity, immobility, and the need to cooperate are missing. These wider trends can be linked to the finding that the journey to school often did not involve neighbourhoods working together. Instead individual caregivers were often the only ones to regularly supervise the journey to school. It was uncommon for caregivers in neighbourhoods to share the supervision of the journey to school. Even two of the three WSBs, which are commonly promoted as a community building exercise (for example, O'Fallon, 2003), involved just one caregiver each. 
So far I have discussed what can be seen to lie behind the journey to school through a comparison of a number of different social and cultural dimensions. I have illustrated how the common lack of distinction that I found between WSBs and other modes of travel to school can be related to four over-arching themes, namely parenting culture, work commitments, risk, and social fragmentation. Through my epistemology I am able to conceptualise these themes, or influences as part of a wider social and cultural system behind the journey to school.

\section{Social and Cultural Influences on Caregivers can be in Conflict}

Conflict between the influences shaping the journey to school were found in the comparisons undertaken in this study and are described by Brittan (1996) as inevitable, especially as society becomes increasingly complex in it's organisation. For example, sometimes I found a conflict of pressures between the priority given to work commitments and the priority given to children. The widespread ideas promoting hard work and ideas stressing the importance of caregivers doing as much as they can to give their child's development a head start can come into conflict. Such conflict arises because working long hours restricts the time available for their children. In the face of such conflicts the outcomes are not always entirely welcome. For example, some WSB caregivers commented that they missed the days before the WSB and the quality one-on-one time they had with their children in the journey to school. Some process seems to be going on so that caregivers respond to the pressures in a way that compromises the time spent with their children. It becomes apparent that it is the social and cultural system that helps caregivers navigate through the conflicts. For example, it is the social and cultural system that steers caregivers towards giving primacy to work commitments and other things, to the expense of quality one-on-one time with their children.

\section{Organising the Conflict}

The social and cultural system over-arching the journey to school helps caregivers navigate through the potential turbulence as smoothly as possible (Douglas \& Wildavsky, 1982). The social and cultural system can be seen to provide a type of 
autopilot for much of what people do. This autopilot goes someway to explain one caregiver's comment about the potential conflict between work and children. At the end of an interview with one caregiver I was asked to describe what sort of things I had uncovered in the project so far. In response to my suggestion about the existence of such potential conflicts the caregiver commented, “... you know but I'd never actually thought of it that way. It's sad though isn't it...because I think of my work and I think that if I don't do it now then I'll have to make it up another time. But I don't think about the time that I spend with my kids in the same way." Through this example it can be seen that the influences or constraints on caregivers' wider lives are organised so that they are not dwelt upon so as to be unmanageable. It is through the organisational processes of the social and cultural system that I commonly found work commitments were a priority around which other aspects of the journey to school were organised. For example, one caregiver described that the journey to school "is primarily focused on benefits for us adults rather than the child's interests." This caregiver acknowledged that such a situation was tough on the child "... but he knows it's just not possible because of the hours I work and the fact that ... it's easier for me to take the car in." The social and cultural system helps caregivers reconcile conflicting constraints as much as possible.

\section{Innovation is Possible}

The over-arching social and cultural system can be seen as an organic web that guides what caregivers do. This social and cultural system lies behind the many commonalities and the lack of distinction between the contexts of both WSBs and other modes of travelling to school. The over-arching system is not rigid. Ideas of

parenting culture and pressures of work overlap allowing caregivers to fit the journey to school around work, while fulfilling the need to be a pro-active caregiver. Driving can be seen to provide evidence of good parenting, and maternal input to familial welfare can be measured by kilometres driven in the journey to school (Sanger, 1995 in Kearns et al., 2003). For example, one non-WSB caregiver commented that it was their role as a good parent to make the journey to school as easy as possible and that this involved driving their children to school. Alternatively WSB caregivers may see 
themselves as fulfilling the role of a good caregiver while coping with the pressures of work by getting involved with a WSB. It becomes apparent that the social and cultural system forms some commonly held guidelines about what is possible in the journey to school and these are then manipulated to varying degrees to suit caregivers' particular situations resulting in different modes of travel. This room for some variation provides an explanation for some differences to be found in comparing the contexts of different journeys to school. 


\section{Chapter Six}

\section{Conclusion}

\section{Limitations and Future Research}

As part of concluding what this research has achieved it is necessary to first give some discussion to what has not been achieved and in doing so acknowledge the limitations of this paper. A general criticism can be related to Lofland and Lofland's (1995) caution that it is difficult to capture and present a dynamic reality in a static text. Faced with extraordinary subtleties and complexity it is not possible for any research to be entirely comprehensive or complete. It is useful to emphasis the above point and that this paper is just a snapshot of the big picture and one interpretation of the journey to school environment. It seems that this paper has raised as many questions as it has provided answers.

A limitation of this research is that the focus has not been on what lies behind the emergence of WSBs per se. In other words, although this research has uncovered that there are a number of over-arching influences, the focus has not sought to establish how particular social and cultural contexts shape the use of particular modes of travel. This would require establishing relationships between specific backgrounds and particular modes of travel. Further research may be able to establish how particular social and cultural ideas are found in particular settings and how these shape different modes of travel. For example, it may become apparent that a relationship exists between proximity of school community to the central business district and caregiver ideas that walking is a favoured mode of travel. This research does little to suggest how the over-arching social and cultural ideas are translated and adopted to suit different caregivers' situations. As such I have not been able to suggest what social and cultural factors lead to the particular modes of travel.

Due to relying on ten interviews for the research this paper relies on a narrow range of samples on which to develop theories. A convenience sample of ten caregivers 
does not do much to cover the multitude of situations in which caregivers live. It has not been necessary to sample a large and representative group of caregivers because the research focus has been on exploring caregiver ideas behind the journey to school and variations in their situation are not significant to this generally applicable question. With further research the context to different modes of travel could be compared over a variety of settings.

The temporal scope of this research is limited, as attention has not been given to how the social and cultural context of the different modes of travel changes over time. This research did not distinguish between caregivers on the basis of the age of caregivers' children and without such controls the children ranged in age from five to thirteen. Such a range of ages introduces variation that results from children being at quite different stages of their development. With this lack of age control it is unclear whether the variation results from age or mode of travel. Further research could investigate how caregivers' social and cultural ideas about the journey to school change over time. Another issue that could be considered by giving greater attention to time would be to investigate whether with more time, WSBs become part of a more distinct social and cultural milieu.

A further shortfall of this research is that it does not provide much in the way of providing directly applicable recommendations for environmentally managing the journey to school. This limitation relates back to the non-action based research focus for this paper as established from the outset. Nonetheless this research raises a number of issues that may be of interest to stakeholders involved in managing the journey to school and the environmental movement more generally. As described by Clarke (2002) environmentalists often have conventionally sought to address environmental issues by amending the underlying social and cultural system. However, such a task is daunting and progress has been incremental at best. Instead Clarke (2002) suggests it is more effective for the environmental movement to focus on changing people's actions rather than changing their underlying beliefs. It is possible that WSBs testify the merits of this approach. Recall that WSBs are a 
rapidly spreading phenomena that involve an environmentally friendly change in people's actions. However, the many similarities found in this paper between WSB and non-WSB caregivers suggests that an underlying change in people's beliefs is not required for this change in action. Other issues include, what hope is there for those people who are less than happy that the pervasive social and cultural system is healthy? Also, if wider things control children's journeys to school and caregivers, what then is at the centre of our lives? These are some of the many issues that this paper has not focused on and these research limitations invite further study.

Another limitation with this research results from difficulties finding enough caregivers involved with WSBs to work with. Having selected two schools I came to discover that both of the WSBs at one of these schools were not what I had initially envisaged WSBs were like. One of the WSBs that I worked with only operated one day per week and not at all during the winter term, due to the cold weather. The other WSB sometimes (it was unclear from the interviews how often this occurred) involved children from only one household, and on these occasions the children from the other household would make their own way to school. Further, both of these WSBs involved always being driven by the same caregiver. Through seeing this variation in WSBs I began to see the picture that I had built up through the literature on WSBs slipping away. This distinction was further undermined when it became apparent how similar an arrangement held by a non-WSB caregiver with his/her neighbour was to what was recognised in other situations as a WSB. It is apparent that the WSB is an idiosyncratic affair with a variety of arrangements. With further research it may be possible to clarify what a WSB is and how they involve a significant degree of variation.

It is granted that having a different and/or broader research focus could have produced a richer set of findings. However, this research focus did not require controlling the sample in terms of social setting or age of children. Neither did it require controlling the sample in terms of social and cultural change over time. The acknowledgement of the above limitations does not undermine what this research has 
achieved. Having described the limitations of this research, it is now appropriate to consider what has been achieved.

\section{Achievements}

This paper aimed to explore a range of social and cultural dimensions of the journey to school involving WSBs and other modes of travel. In-depth interviews were held with ten caregivers and from these discussions I identified four themes, in particular caregiver ideas about their role, the work commitments that caregivers have, the risks posed by strangers and traffic, and the social fragmentation of neighbourhoods. With the exception of work commitments, Kearns et al. (2003) and Collins and Kearns (2001) also identified these themes as important aspects of the journey to school context. The themes were commonly identified by caregivers and have been found to be significant dimensions of the social and cultural background of different modes of travel. Recall that in Chapter five some description was given into how these over-arching themes are infused into different modes of travel. However, as recognised in the limitations section above, the focus of this paper is not on how particular social and cultural ideas are translated into the different modes of travel. This discussion was only expanded in so far as to further establish that such a system exists.

The second aim of this paper was to compare and contrast some key social and cultural dimensions of WSBs and other modes of travel. Through this comparison I found that the social and cultural background for different modes of travel shared many similarities. It is apparent that the journey to school is only part of caregivers' wider lives. Further, caregivers live in the same society and are moulded by similar influences.

Due to the importance of seeing the journey to school amongst a web of wider constraints I propose that rather than describing it as a space it is more descriptive to conceive of it as an interstice. In other words, it is something that is shaped by wider influences. It is the something-in-the-middle that is shaped by what occurs either 
prior to or following it. The term space involves connotations that something is actively shaped. However, the journey to school is something that is very much embedded in a wider context and is a marginalised part of such processes. As a consequence of the journey to school being marginalised, children are also marginalised and this is a finding that is supported by Kearns et al. (2003).

The existence of a wider social and cultural system does not categorise individuals as insignificant or inadequate. Instead I suggest that individuals are to be recognised as important as well. Douglas and Wildavsky (1982) usefully suggest that the social and cultural system is to be viewed as a valuable resource that helps individuals organise their environment. Remember as introduced in Chapter two the social and cultural viewfinder used in this paper views that individuals have some degree of agency within the system. So I am not suggesting that individuals are weak for being manipulated. Rather individuals somehow adapt the over-arching guiding influences.

As individuals are members of wider communities and systems it is unfair and misleading to scapegoat them when we do not like what is happening and we want to manage some change. As Jacobs (1961) suggest, it is simplistic to view cars as the source of problems in city planning. Instead we need to recognise the systemic nature of the issue. Having found that a similar system of organisation underlies the journey to school, I propose that this issue is also conceptualised as part of something wider. I acknowledge, however, that this suggestion on how to look at the journey to school may involve challenging the reader's underlying ideas. For example, the reader may be more adept at understanding the journey to school by paying more attention to the physical components and less to the wider social and cultural system. As suggested by Clarke (2002) though, it is unclear how effective it is trying to change people's underlying ideas. So I recognise that the argument developed in this paper is limited and that it's ability to change the reader's ideas needs to be kept in perspective. 
Returning to Kearns et al.'s (2003) study on WSBs it is possible to illustrate how the findings of this paper compliment their findings. Recall that Kearns et al. (2003) concluded that WSB provide little distinction from motorised modes of travel in terms of young people re-claiming the streets and challenging the caregiver practice of escorting their children to school. Kearns et al. (2003) considered a wide range of social and cultural issues that lie behind the journey to school. Unlike Kearns et al. (2003) this research takes an in-depth look at what lies behind both WSB and nonWSBs. As such an explanation is precipitated for Kearns et al.'s (2003) conclusion. By recognising WSBs and other modes of travel are part of an over-arching system of social and cultural influences it is unsurprising that WSBs do not provide a radical break from other modes of travel. 


\section{References}

Baddeley, S. (2000) Baddeley on "the school run."

http://www.ecoplan.org/children/ch_index.htm (accessed 19 February 2003).

Baalbergen, J.R. (2004) 'Walking School Busses are here to stay - Tizard.'

Environmental News, 1(3).

Beck, U. (1992) Risk Society: Towards a New Modernity. London: Sage

Publications.

Beck-Gernsheim, E. (1996) Life as a Planning Project. In Lash, S., Szerszynski, B., and Wynne, B. (Eds.) Risk, Environment and Modernity: Towards a New Ecology. London: Sage Publications, pp. 139- 153.

Bernard, H.R. (2002) Research Methods in Anthropology: Qualitative and Quantitative Approaches. $3^{\text {rd }}$ Ed. Altamira Press: Oxford.

Bickerstaff, K. and Shaw, S. (2000) An Evaluation of the Walking School Bus at Pirehill First School. A report commissioned by The Staffordshire Walking Bus Partnership.

Black, C., Collins, A., and Snell, M. (2001) 'Encouraging Walking: The Case of Journey-to-school Trips in Compact Urban Areas.' Urban Studies 38(7): 1121-1141.

Bourdieu, P. (2003) 'Participant Objectivation' Journal of the Royal anthropological Institute. 9: 281-294.

Bradburn, N.M. (1983) 'Response effects' In Rossi, P.H., Wright, J.D., and Anderson, A.B. (Eds.) Handbook of survey research. New York: Academic Press, pp. 289-328.

Brittan, A. (1996) 'Social structure and structuration.' In A\& J Kuper (Eds.) Social Science Encyclopedia ( $2^{\text {nd }}$ Ed.) London: Routledge, 803- 805.

Britton, E. (2000) Opening Remarks. http://www.ecoplan.org/children/ch_index.htm (accessed 19 February 2003).

Clarke, J. (2002) Secrets of the Darkside Keynote address for the New Zealand Environmental Education Association conference. http://www.nzaee.org.nz/conferences/papers/Jason\%20Clarke.doc (accessed 
05 December).

Collins, D.C.A. and Kearns, R.A. (2001) 'The Safe Journeys of an Enterprising School: Negotiating Landscapes of Opportunity and Risk.' Health \& Place 7: 293-306.

Denzin, N.S. and Lincoln, Y.S. (Eds.) (2000) Handbook of Qualitative Research. ( $2^{\text {nd }}$ Ed.) London: Sage Publications.

Department of Labour (2003) What's a "normal” working week? http://www.dol.govt.nz/futureofwork/workplace-normalhours.asp (accessed 02 December)

Department for Transport (2003) School Travel http://www.local-transport.dft.gov.uk/schooltravel/ (accessed 28 March).

Dingwall, R. (1997) 'Accounts, Interviews and Observations.' In Miller, G. and Dingwall, R. (Eds.) Context and Method in Qualitative Research. London: Sage Publications, pp.51-65.

Douglas, M. (1992) Risk and Blame: Essays in cultural theory. London: Routledge.

Douglas, M. and Wildavsky, A. (1982) Risk and Culture: An Essay on the Selection of Technical and Environmental Dangers. London: University of California Press.

Ellen, R.F. (Ed.) (1984) Ethnographic research: A guide to general conduct. London: Academic Press.

Engwicht, D. (1993) Reclaiming our cities and towns: better living with less traffic. Philadelphia: New Society Publishers in cooperation with Envirobook, Australia.

Fielding, N. (Ed.) (2003) Interviewing. London: Sage Publications.

Fertig,G. (1996) 'Investigating the process of culture change from an anthropological perspective.' The Social Studies. Jul/Aug, pp. 165-171.

Fetterman, D.M. (1989) Ethnography: Step by Step. Applied Social Research Methods Series, Vol. 17. London: Sage Publications.

Flick, U. (2002) An Introduction to Qualitative Research. (2 ${ }^{\text {nd }}$ Ed.) London: Sage Publications. 
Fontana, A. and Frey, J.H. (2000) 'The Interview: From Structured Questions to Negotiated Text.' In Denzin, N.S. and Lincoln, Y.S. (Eds.) Handbook of Qualitative Research. (2 ${ }^{\text {nd }}$ Ed.) London: Sage Publications, pp. 645-672.

Geertz, C. (1973) The Interpretation of Cultures: Selected Essays. New York: Basic Books, Inc.

Gibbons, M.T. (Ed.) (1987) Interpreting Politics. Oxford: Basil Blackwell Ltd.

Giddens, A. (1987) Social Theory and Modern Sociology. Cambridge: Polity in association with Blackwell.

Goudie, A. (2001) The Nature of the Environment. ( $4^{\text {th }}$ Ed.) Oxford: Blackwell Publishers.

Hutchby, I. and Wooffit, R. (1998) Conversation Analysis: Principles, Practices and Applications. Cambridge: Polity.

Jacobs, J. (1961) The death and life of great American cities. New York: Vintage Books.

Kearns, R.A., Collins, D.C.A., and Neuwelt, P. (2003) 'The Walking School Bus: Extending children's geographies?' Area 35(3):285-292.

Kearns, R.A. and Collins, D.C.A. (2003) An Assessment of Walking School Buses in the Auckland Region. A Report to: Auckland Regional Council.

Kokotailo, R. (2000) New Zealand Pedestrian Profile: An Overview of Pedestrian Activity \& Injury in New Zealand.

Landry, C. (1995) 'Liveability in the city.' In Greenfield, N. (Ed.) Melbourne: our city, our culture. South Melbourne VIC 3205: Hyland House, pp. 12-31.

Lofland, J. and Lofland, L.H. (1995) Analyzing Social Settings: A Guide to Qualitative Observation and Analysis. $\left(3^{\text {rd }}\right.$ Ed.) Melbourne: Wadsworth Publishing Company.

Mouchel (2003) What is a Walking Bus? http://www.walkingbus.com/index.htm (accessed 25 November).

Newby, H. (1997) 'One World Two Cultures: Sociology and the Environment.' In Owen, L.A. and Unwin, T. (Eds.) Environmental Management: Readings and Case Studies. Oxford: Blackwell Publishers, pp. 471-479.

O'Fallon, C. (2003) The Walking School Bus: A Guide for Parents and Teachers. 
http://www.pinnacleresearch.co.nz/wsb/WSB\%20GUIDEPARENTS.pdf (accessed 19 February).

O'Fallon, C. (2001) Walking School Bus Networks: Evaluation of Trial in Christchurch. http://www.pinnacleresearch.co.nz/WSB\%20trial\%20report\%20June\% 202001.pdf (accessed 04 April 2003).

O'Fallon, C., Sullivan, C., and Hensher, D.A. (2002) Understanding Underlying Constraints Affecting Decision-Making by Morning Car Commuters. Working Paper ITS-WP-02-13. http://www.its.usyd.edu.au/publications/2002_working_papers/ITS-WP-0213.pdf (accessed 23.04.03).

Poland, B. and Pederson, A. (1998) 'Reading Between the Lines: interpreting silences in qualitative research.' Qualitative Inquiry. 4(2):293-313.

Psathas, G. (1995) 'Conversation Analysis: The Study of Talk-in-Interaction'. Qualitative Research Methods, Vol. 35. London: Sage Publications.

Rapport, N. and Overing, J. (2000) Social and Cultural Anthropology: The Key Concepts. London: Routledge.

Rosman, A. and Rubel, P. (1995) The Tapestry of Culture: An Introduction to Cultural Anthropology. Auckland: $\mathrm{M}^{\mathrm{C}} \mathrm{Graw}-H i l l$, Inc.

Sanger, C. (1995) 'Girls and the getaway: cars, culture, and the predicament of gendered space.' University of Pennsylvania Law Review. Vol. 144, pp.705756.

Sangren, P.S. (1992) 'Rhetoric and the authority of ethnography: 'postmodernism' and the social reproduction of texts.' Current Anthropology 33(1): 277-308.

School Transportation Group (2001) An Analysis of North Carolina Guidelines and Criteria for Establishing School Walk Zones. http://itre.ncsu.edu/stg/downloads/FinalReport-SchoolWalkZones.pdf (accessed 07.04.03).

Scoop, 2004 'Time to Protect Kids and the Climate,' 27 January. http://www.scoop.co.nz/mason/stories/PA0401/S00204.htm (accessed 21/02/04). 
Silverman, D. (2000) 'Analyzing Talk and Text.' In Denzin, N.K. and Lincoln, Y.S. (Eds.) (2000) Handbook of Qualitative Research. ( $2^{\text {nd }}$ Ed.) London: Sage Publications.

Solnit, R. (2000) Wanderlust: A History of Walking. London: Viking.

Spradley, J.R. (1979) The ethnographic interview. Fort worth, TX: Harcourt Brace Jovanovich.

Suzuki, D. and Dressel, H. (1999) Naked Ape to Superspecies: A Personal Perspective on Humanity and the Global Eco-Crisis. St Leonards NSW 1590, Allen and Unwin.

Swift, R. (2002) 'Rush to nowhere' New Internationalist 343: 9-12.

ten Have, P. (1999) Doing Conversation Analysis: A Practical Guide. London: Sage Publications.

Tolich, M. and Davidson, C. (1999) Starting Fieldwork: An Introduction to Qualitative Research in New Zealand. Oxford University Press: Auckland.

Weyns, W. (1998) 'The Cultural Filter.' In Nath, B., Hens, L., Compton, P., and Devuyst, D. Environmental Management in Practice: Instruments for Environmental Management. Volume 1. London: Routledge, pp. 44-61.

White, I.D., Mottershead, D.N., and Harrison, S.J. (1992) Environmental Systems: An introductory text. ( $2^{\text {nd }} E d$.) London: Chapman \& Hall. 


\section{Appendix One}

\section{Aide Memoir}

Preamble-Greetings, informal chatter, e.g. the weather, MENVst student, etc.

- Interest in different methods of moving around and why different people use different modes of travel. My particular focus is the journey to school.

- Some similar studies have been done in Christchurch and Auckland and I would like to fill the gap for Wellington.

- Any questions you don't want to answer you don't have to.

- Also please feel free to ask me to clarify anything unclear.

- Could you please sign the consent form?

1. First I would like to find out how many people there are in this household.

- How many children do you have going to school?

- What school(s) do they go to?

- How old are they?

2. Now I'd like to talk about the route. We can use this map as a prop if you'd like.

- What is your address (if I don't know this already)?

- So we are here (point to the map)... and which way does...go to get to school?

- Can you describe the route to me?

- What sorts of things do you pass? E.g. parks, shops, etc.

- What are the roads like?

- Where do they cross? What is it like? Etc.

- What do they do on the way?

- How many people are around?

- Are they walking/driving/biking/etc...?

- Do you know these people? 
- What are they like?

- How long does it take to get to and from school?

- How does this route compare to this...(pointing to alternative on the map) route?

- Does the weather affect how your children get to school? What do you do? Why?

- What do you like about this route?

- What do you not like about this route?

3. Now lets focus on how your child gets to school?

- Do they use a car, bike, bus, walk, etc?

- Have your children always used this mode?

- How have you changed what you use?

- How many cars do you have in the family?

- What type of car do you have? E.g. is it a small car, 4WD, van, people mover, etc?

- How many bikes are available?

- What choices are there in how your child travels to school?

- What made you decided not to use these?

- What are the benefits of taking the car/WSB/etc?

- What are the drawbacks/downsides/costs?

- What are the important things or priorities that need to be considered?

4. Next I'd like to ask you a few questions about who's involved in getting the child to school.

- Who gets the children ready to leave in the morning?

- Who meets them in the afternoon?

- Who takes them/picks them up?

- Do they go all the way to the school gate or somewhere midway (e.g. a bus-stop, or to meet friends)? 
- How did you choose... involved as opposed to someone else?

- What responsibilities are involved?

- What happens if the person who usually takes the child to or from school can't?

- Who decides how your child gets to school?

- $\quad$ How was this decision made?

5. Now I'd like to talk about how your decision to walk/drive/etc. fits your lifestyle?

- $\quad$ Did you walk to school?

- What has changed (that prevents your child(ren) from walking)?

- How often do you walk/drive/etc?

- What affects this choice?

- In what situations?

- $\quad$ How do different situations affect you choice?

6. Finally I'd like to complete this personal information section.

- Which ethnic group do you identify with?

- What is your age?

- What occupations do the adult members of your household have?

- Are these full/part time?

- In which bracket does your household income fit? [ $<\$ 20000]$ [\$20 000-\$39 999] [\$40 000-\$59 999] [\$60 000-\$79 999] [\$80 000-\$100 000] [>\$100 000]

Thank you very much. Please contact me if there's something that you have any further questions or comments. 


\section{Appendix Two}

\section{Coded Interview Data}

\section{Comments Reflecting Caregiver Ideas About Environmental Value for Children}

\subsection{WSB}

\subsubsection{Caregiver A}

It is positive being able to be outdoors. The journey to school provides an opportunity for his/her boy to experience the environment in a hands-on way. "Stopping and looking at things along the way... finding dead insects and... you know looking at trees... and noticing the flowers coming out and stuff." Caregiver A believes that "kids like being outdoors in the environment and being able to find trees shaped like dragons." Caregiver A talks favourably about "being in the community rather than in a car all the time." Caregiver A describes the scenery along the walk as "pleasant, pretty, and green".

\subsubsection{Caregiver $B$}

Describes it as positive how the journey to school provides an opportunity for his/her son to "stop and look at a spider on the way and that kind of stuff."

\subsubsection{Caregiver $\mathrm{C}$}

Recognises the value of fresh air that the children get to experience while walking in the journey to school. It is also "healthy to be outdoors spotting things and being able to have a hands-on experience."

\subsubsection{Caregiver $D$}

Although Caregiver D thinks that there is not much time to stop at specific sites along the way, like a park, there is however, value in just being outdoors in the environment. Passing through the environment on foot and having a hands-on experience is recognised as having value. For example, "the children can explore the plants and animals, such as collecting pinecones and wetas." It also provides an opportunity to see and think about the social activities going on around the children, such as "what are those people doing?" 


\subsection{Non-WSB}

\subsubsection{Caregiver $\mathbf{E}$}

Acknowledges that by taking the car his/her son misses out on the hands-on experience of being able to walk. Caregiver E recalls that being out in the environment on your own two feet and, for example, being able to "walk along to school and kick a stone, etc." was an important part of their own childhood and development.

\subsubsection{Caregiver $F$}

The journey to school environment is just something that needs to be crossed on the way to school.

\subsubsection{Caregiver $\mathbf{G}$}

Caregiver $\mathrm{G}$ walks to school most of the time and they enjoy at least some parts of the environment, which is travelled through. Caregiver G speaks unfavourably of the "yukky, muddy, and wet" places. However, Caregiver G seems to accept these detractions hand-in-hand with the positives. Caregiver G likes simply being able to spend time outdoors "...to notice the nice plants... or find a dead bird". Caregiver G thinks that it is positive to "appreciate, connect, and learn from the environment."

\subsubsection{Caregiver $H$}

Identifies environmental appreciation as an important part of the journey to school.

\subsubsection{Caregiver I}

Recognises the value of being able to "ground" oneself in the immediate environment as part of the journey to school.

\subsubsection{Caregiver $\mathbf{J}$}

Recognises that walking permits his/her boys to be "outside in the environment and allows them to learn to appreciate it". Caregiver J feels this is a good thing to develop. 


\section{Caregivers Ideas Regarding the Types and Quantities of People in the Journey to School and the Value of Meeting them.}

\subsection{WSB}

\subsubsection{Caregiver A}

Sees lots of people in the journey to school space. Mostly these people seem to be adults walking to work. However, there are a couple of children walking to school that he/she sees. Caregiver A guesses that "most children are being driven for the journey to school." Caregiver A mentions the importance of being safe from people as well as safe from traffic. Caregiver A suggests that he/she is "not particularly paranoid in regards to stranger danger and there is not a problem, we are after all social beings." However, some risk is still identified with strangers. Caregiver A likes it, that the journey to school allows the children to participate in community building, such as seeing and meeting people.

\subsubsection{Caregiver $B$}

Comments that the journey to school involves a busy pedestrian environment. Many of these pedestrians seem to be tertiary students or are children being walked to school. Caregiver B mentions that he/she knows a lot of people living in the community on the journey to school. His/her son could call on this community in a situation of trouble. Caregiver B makes no direct reference to stranger danger. Being social in the journey to school is a positive element for both caregivers and children. The journey to school reinforces relationships between people. Caregiver B believes that the social aspects of WSB's have a drawback due to the hassle of being responsible for organising people. Caregiver B recognises that the journey to school provides an opportunity for both "kids and caregivers to make friendships."

\subsubsection{Caregiver $\mathrm{C}$}

Describes that most people they see are in cars. Other people are waiting at bus stops and there are small numbers of children walking to other schools. Caregiver $\mathrm{C}$ mentions that the journey to school is in an area that is not safe for young children to 
walk around unsupervised. Being out on the street meeting people and community cohesion is valuable.

\subsubsection{Caregiver $D$}

Views other people in the journey to school space as posing a potential safety risk. Caregiver D perceives that there are lots of people "rushing around doing crazy things and they are not to be trusted," especially in regards to children. Caregiver D observes that there are lots of other children in the journey to school space and that there are many commuters going to work. Caregiver D mentions having not directly seen the dodgy people, even though he/she sees lots of people. Nonetheless

Caregiver D feels threatened that all the horrid things that he/she hears about in the media could happen to someone they know. Caregiver D commented that he/she knows a lot of people in the community along the journey to school route. Caregiver D suggests that community building is valuable and that building a safe community "helps to allow children to play on the street."

\subsection{Non-WSB}

\subsubsection{Caregiver $\mathbf{E}$}

Recognises that his/her child's journey to school involves passing lots of people, including numerous schools and school pupils walking to school. Sometimes from the car they see pupils from the same school waiting at the bus stop so they stop and give them a lift. Caregiver E observes that there are lots of people walking.

Caregiver E perceives a stranger danger risk in the journey to school. Not being able to walk home and live in the school's local community is perceived by Caregiver $\mathrm{E}$ to be a negative element of their journey to school.

\subsubsection{Caregiver $\mathbf{F}$}

Caregiver F notices that around a suburban shopping centre on the route to school there are some people out on the street. On the whole though, Caregiver F perceives that few people use walking to get places. Although Caregiver F has never experienced any dangers in the journey to school, he/she perceives that there is a risk. Caregiver F comments that risks surround more than just people who he/she does not know and "dangers can surround people who you know." 


\subsubsection{Caregiver $\mathbf{G}$}

Mentions seeing about 4-5 people in the short space of time that they are on the journey to school. These people are described as people going places. The journey to school route is described as "a bit of a thoroughfare for people walking." Caregiver G knows only a few of the people he/she sees. However, "the large number of people going through the area poses a bit of a safety issue." Caregiver $\mathrm{G}$ is under the impression that "every school has got a lurker" and that the journey to school is prime territory for such a person. This perception is linked to discussions with his/her friends and what is portrayed in the media. Caregiver G does not "like the idea of even being around such a person," even if they are not and never will be a danger. Caregiver $\mathrm{G}$ does not know many other people in the journey to school community.

\subsubsection{Caregiver $\mathbf{H}$}

\section{$\mathrm{N} / \mathrm{A}$}

\subsubsection{Caregiver I}

First Caregiver I suggests that he/she does not really take much notice of the number or types of people they pass in the journey to school. Caregiver I identifies some of the walkers as students, which he/she describes in a harmless way as "floating around." Also Caregiver I notices other children walking to school. Caregiver I knows quite a few of these children. A positive element of the journey to school is seeing and meeting people. Being social is described as being a positive part of walking for the journey to school. Walking with his/her children to school assists Caregiver I to be a closer part of the classroom as he/she can easily come into the school grounds, especially as they have to pass through the area en route to work. Walking also helps bond both children and caregivers as part of the community. Caregiver I perceives that a stranger danger does exist.

\subsubsection{Caregiver $\mathbf{J}$}

The journey to school occurs where other people can see Caregiver J's boys.

Caregiver J perceives that "there are always people on the street for both the journey to and from school and this provides safety" through people being able to watch out for the children. Caregiver J's children are not allowed to meet up with anyone 
during the journey to school but this is so they will not arrive to school late.

Caregiver J perceives that public spaces with lots of people in them are safer than those, which are quiet. It is in these isolated spaces where stranger danger is an issue. Caregiver $\mathrm{J}$ recognises that a lot of people in the community know him/her and his/her boys.

\section{Caregiver Supervision and Responsibilities During the Journey to School}

\subsection{WSB}

\subsubsection{Caregiver $\mathbf{A}$}

The WSB has some rules, such as "everyone has to stop and check before crossing a road." The children decide whether to cross or not and the adult is responsible for supervising their judgement. So the building up of road awareness is part of the supervision. Caregiver A feels that the children are sensible though. Caregiver A "liked the idea of a WSB and felt responsible to support and make it more accessible to others." The journey to school provides a valuable opportunity for children to "get some fresh air and exercise before settling into class." Using a WSB for the journey to school provides an opportunity for children to develop good habits, such as being able to walk rather than relying on a car. The journey to school also provides an opportunity to build a community. As a caregiver, Caregiver A feels some responsibility that the journey to school is safe in terms of traffic and strangers and that the practicalities, such as getting to school on time, are met. Caregiver A feels responsible for waiting with his/her son until the WSB arrives. Caregiver A describes how children in the journey to school also have responsibilities and it is not just with the caregivers. For example, there are certain rules that children must abide by to be part of the WSB. The journey to school also involves social supervision from the caregivers, for example overseeing that the children are behaving well with one another. This element is informal though and is described as just being a matter of "chatting with the children." Caregiver A would not allow his/her son to go on the journey to school by himself until he is about ten years old. This age limit is required 
to develop road awareness and in terms of experience to cope with potential stranger danger.

\subsubsection{Caregiver $B$}

A parent's role is to supervise their children, which Caregiver B spelt out to me as being a matter of "super-vision... keeping an eye over children." Caregiver B described the journey to school as being reasonably relaxed and there would be no liability suits from other caregivers involved in the WSB if something were to happen. The journey to school is presented as a "casual affair" that is flexible enough to accommodate the complexity and need for spontaneity in life. However, the journey to school involves some structure so as to provide a middle ground on which the group can come together. For example, some independence is given to the children so that "they can stop and pick up insects if they want or they can go faster if they would like to get to school in a hurry. Though such flexibility is still within the constraints of getting to school on time." Having a uniform and regimented journey to school does not fit those involved. Note that the school has no uniform either. While Caregiver B describes the journey to school as quite relaxed he/she acknowledges that some rules do exist, for example "everyone stops and waits until they are all together where they need to cross a road." Caregiver B believes that the journey to school provides an opportunity to develop in children the healthy habit of walking to get places rather than becoming reliant on sitting in a car. At six years old Caregiver B's son needs supervision because he has an inadequate level of road sense. This might change by the age of 9 or 10 . Road safety is the main issue. No unique skills are required to supervise the WSB, except that the caregiver is required to be organised. Note that Caregiver B was age 9 or 10 when they gained independence in their own journey to school as a child. The journey to school provides a valuable opportunity for kids to run and play. Allowing kids an opportunity to get some "fresh air and exercise is valuable before getting them to sit inside during class. Burning a bit of energy first helps them to concentrate."

\subsubsection{Caregiver $\mathbf{C}$}

The journey to school provides an opportunity to teach children about road safety. The WSB also provides community education so that the wider community develops 
awareness that walking is possible and that they are to be aware of kids in their journey to school. The journey to school provides Caregiver $\mathrm{C}$ with a good "opportunity to mingle with the kids." An important responsibility for Caregiver $\mathrm{C}$ in the journey to school is ensuring his/her children's safety in regards to stranger danger and road safety. Caregiver $\mathrm{C}$ also sees that providing an opportunity for exercise is important and that it is valuable to get the children into this healthy daily routine. Caregiver $\mathrm{C}$ thinks that "by the age of 10 or 11 the children should be able to walk to school by themselves, or alternatively they should be able to catch the bus."

\subsubsection{Caregiver D}

For the journey to school Caregiver D thinks it is positive to supervise children in response to stranger danger. Sometimes some of the relatively old children who come along with the WSB do not want to hang out with the younger ones. In this situation Caregiver D pays attention to looking after the young ones and keeps less of an eye on the older ones. The WSB with all its flags and sashes provides an opportunity to increase community awareness about walking as a mode of transport and getting people to look out for children on their journey to school. The journey to school provides a good opportunity for exercise and an opportunity for children to learn about traffic safety and about stranger danger safety. The journey to school also provides an opportunity to ask, explore, and learn about the environment around the children. Caregiver D was prompted to get involved with the WSB for the journey to school because he/she wanted to help the children in the house across the road from his/her house to become less dependent on the car for getting to school. Caregiver D was also prompted by the numerous efforts by the school encouraging healthy eating habits. These efforts involved looking at adopting a broad range of lifestyle changes. WSBs for the journey to school are a way of developing independence in children, which is rewarding for them. About once a week Caregiver D lets his/her 8 and 11year-old children go by themselves to school when he/she is busy with the younger one. The older children have learnt a safe routine for the journey to school. Caregiver $\mathrm{D}$ is not entirely comfortable with this though and talks about a case in which a 17 year old was picked up by strangers. Caregiver D would never let one of his/her 
children walk home by themselves. As a driver of the WSB, Caregiver D has a responsibility in regards to teaching traffic safety.

\subsection{Non-WSB}

\subsubsection{Caregiver $\mathbf{E}$}

Supervising the safety of his/her son in terms of stranger danger and traffic danger is a responsibility for Caregiver E. This supervision, Caregiver E acknowledges, is "primarily focused on benefits for us adults rather than the child's interests." Caregiver $E$ believes that because of this arrangement that suits the adults, the child misses out on a number of valuable experiences, such as being able to "walk along to school and kick a stone, etc." Caregiver E suggests that having a space for the child in the journey to school is an important experience for them and helps get them "psyched up for the day at school." Caregiver E laments that his/her son misses out on this because they get a lift in the car. An important aspect of supervising children is that "it fits with everybody's schedule," especially with the adults. Under Caregiver E's supervision he/she gets their child as close as possible to their school supervision space, such as right inside the gate and up to the classroom. There is a fear that his/her son will get mislead or distracted en route. Caregiver E feels that his/her son is not old enough to catch the bus to school without caregiver supervision. This is especially the case if Caregiver E's son catches the bus with a friend as this creates worries about "the boys getting up to mischief." However, Caregiver E's son feels that "catching the bus is a treat." Caregiver E recognises that his/her son is "ready for being given some more responsibility." Caregiver E is not confident with his/her son being able to cross the road safely. A key responsibility for Caregiver $\mathrm{E}$ in the journey to school is making sure that the vehicle is ready, e.g. has a full take of fuel. Caregiver E recognises that all members of the family have got their own responsibilities so that the journey to school goes to plan. Caregiver E feels that his/her son gets sufficient exercise with soccer and other school activities without having to do more exercise in the journey to school.

\subsubsection{Caregiver $\mathbf{F}$}


Keeping the children in sight at all times is not necessary for Caregiver $\mathrm{F}$ as they are allowed to walk through the park by themselves. In his/her previous house Caregiver F used to let his/her 6 year old daughter walk to school. Caregiver F's two children now go to the same school and Caregiver F has let them walk from the bus stop to school for the last four months. "The two children keep an eye out for each other." A major prompt for Caregiver F to let the children make their own way to school has been advise from a teacher who suggested that the children needed to be given opportunities to develop some more independence. As Caregiver F's parents did not have a car he/she had to walk all the time when they went to school. Although it was not far, Caregiver F still seems to resent that having to walk while other kids were dropped off to school by car. Caregiver F feels that it is necessary to make the journey to school as easy for children as possible.

\subsubsection{Caregiver $\mathbf{G}$}

Caregiver G describes him/herself as being solely responsible for his/her son.

Caregiver G describes his/her role in regards to road safety as supervisory. His/her son has developed what Caregiver $\mathrm{G}$ feels is a good safety awareness in terms of road safety. Caregiver $\mathrm{G}$ describes that he/she likes the walk to school and that it is a good thing. Caregiver $\mathrm{G}$ wants to develop in his/her son a positive attitude towards walking and appreciating the environment. Walking to school provides his/her son with an opportunity to be invigorated before sitting in class. Caregiver $\mathrm{G}$ needs to supervise his/her son so that the journey to school fits his/her timetable, which is a key priority. Caregiver supervision of the journey to school is described by Caregiver $\mathrm{G}$ to be chiefly concerned with making sure that it is safe.

\subsubsection{Caregiver $\mathbf{H}$}

Caregiver $\mathrm{H}$ likes the idea that his/her children are supervised during the journey to school, such as when they were part of a WSB. This way the children learn about road safety for the journey to school. Caregiver $\mathrm{H}$ would like his/her children to walk to school while being supervised. However, Caregiver $\mathrm{H}$ feels that his/her children have become too used to the pattern of being dropped off to school in a vehicle. Caregiver $\mathrm{H}$ describes that some of his/her older children are able to look after themselves in the journey to school. Although Caregiver $\mathrm{H}$ would have loved his/her 
children to walk to school, this is not a priority. Caregiver $\mathrm{H}$ feels that his/her children have alternative sources of physical activity to keep them fit and healthy. Caregiver H describes "walking is a good habit." Making sure that his/her children are on time and have what they need for school is an important responsibility for caregivers and not children. Without supervision Caregiver $\mathrm{H}$ does not trust where his/her children might get to on the way to school. It is not possible for Caregiver H's older children to keep an eye out for the younger children, as the older son does not want to be seen with his younger siblings.

\subsubsection{Caregiver $I$}

Caregiver I has let his/her 9-year-old daughter do the journey to school by herself once, but he/she certainly would not allow his/her five-year-old son to do this.

Caregiver I describes his/her daughter as careful but her son does not have the road sense and will not be able to do the journey to school by himself for a few years yet. "Maybe this will be possible when he is eight." Before his/her son started school a few months ago Caregiver I started letting his/her daughter walk part of the journey to school with a friend. However, this no longer happens because they all walk together. At the moment his/her five-year-old son is not mature or aware enough to do the journey to school unsupervised. Even with Caregiver I's two children walking the journey to school together they will not be able to do it by themselves for a couple of years yet and, even then, they would still need to be supervised across the main busy road before being able to do the rest of the journey to school unsupervised. Walking to school provides a good opportunity for healthy exercise as well as the prospect of walking being incorporated into the children's daily routine. Supervision is needed primarily in terms of traffic but also in terms of stranger danger. Walking with his/her children as to school provides an opportunity for the caregivers to become more part of what is going on in terms of their children's school activities. Caregiver I is comfortable that his/her partner and their neighbour all have the skills required to supervise the journey to school "These skills are those that are common for looking after children of that age." People with these skills are not unique. Key responsibilities in the journey to school are what Caregiver I 
describes as "the practicalities, such as leaving on time and holding their hand while crossing the road."

\subsubsection{Caregiver J}

Caregiver J's children are able to look after each other in the journey to school. An important supervisory role for caregivers is to look out for where the children are walking and to make sure that it is within public spaces where there are many eyes on the look out.

\section{Transport and Equipment Available}

\subsection{WSB}

\subsubsection{Caregiver $A$}

Prior to the WSB being available, Caregiver A was already walking to school. Caregiver A's household has recently got a second car. However, the recent acquisition does not get used except for holidays. A bike is available for the journey to school. However, biking is not an option because "the road is too busy and the hills are too much of an effort." Caregiver A's son used to use a scooter to get to school on but having become part of the WSB this is no longer possible. Caregiver A believes that "it don't make good sense to drive and it is good to walk."

\subsubsection{Caregiver $B$}

There is one small car in the household. Bikes are available for the journey to school. However, Caregiver B's child is not competent enough to ride the bike to school.

\subsubsection{Caregiver $\mathrm{C}$}

There is one car in the household. Caregiver $\mathrm{C}$ also has a pushchair for his/her preschooler so they can all walk together. No bikes are available for the journey to school. Using "the car is very convenient and it is easy to get into the habit of using the car for lots of things." Caregiver C drives more than he/she wishes to. Driving is quicker, Caregiver $\mathrm{C}$ can carry much more gear than he/she would otherwise be able to, it does not get wet when it rains, and you can go further.

\subsubsection{Caregiver D}

Caregiver D has two flags and two sashes for the WSB. Caregiver D's children have always walked to school. Caregiver D does not have a car available for the journey 
to school. Caregiver D's children have bikes, but he/she is concerned about the bikes being stolen. Also his/her children need to develop greater road awareness through walking before they will be competent enough to bike. The WSB does not cost anything.

\subsection{Non-WSB}

\subsubsection{Caregiver $\mathbf{E}$}

A large vehicle is used all the time for the journey to school. Note that when they used to live closer to school they used to walk. As there is only one car available for the journey to school, its availability has a big affect on the journey to school. A couple of times it has been in at the mechanics and they have used the bus for the journey to school. Note that the bus departs from right outside their house. Caregiver E describes that the bus just does not suit their timetable and it involves swapping buses. There are low costs involved in running the vehicle and "it runs on the smell of an oily rag." Caregiver E's son has a bike. However, Caregiver E feels "it is too far to bike to school and [his/her son] has the confidence but not competence in terms of road sense." Caregiver E suggests that because the route that they drive to school meets up with the WSB, which they sometimes see, it is possible they could join the WSB for getting to school. However, this has not occurred because Caregiver $\mathrm{E}$ has not got in contact with the people organising the WSB who Caregiver E does not know. Further Caregiver E's son does not want to "hang out with the younger ones who are involved in the WSB." Sometimes when Caregiver E's partner has time off he/she takes their son to school on the bus. If they changed their son to the local school there is a school bus available and this would only take about 20 minutes. However they are "very happy and settled" at the existing school and do not want to upset this arrangement. Maybe next year Caregiver E will let his/her son walk unsupervised for part of the journey to school, but at the moment Caregiver $\mathrm{E}$ and his/her partner do not think that their son is old enough. Caregiver E feels that it is time that his/her son starts to be given some more responsibility, as he is ready for this.

\subsubsection{Caregiver $F$}


Caregiver F only drives his/her children to school when he/she is not working.

Caregiver F used to work five days a week so they always caught the bus. However, the arrangement had changed from the week that I held the interview onwards so that Caregiver F only worked three days a week. There is one car in the household in which they all fit. Buses are handy for the journey to school and subsequent work commitments.

\subsubsection{Caregiver $\mathbf{G}$}

There are two cars in the household. A bike is available for the journey to school. However, this is not used, as it is impractical to ride the route. Wet weather gear is available.

\subsubsection{Caregiver $\mathrm{H}$}

Caregiver $\mathrm{H}$ would prefer his/her children to walk to school. However, Caregiver $\mathrm{H}$ explains that as he/she is coming down to school anyway for day-care for his/her "little ones" he/she does not feel able to make the other children walk. "It is easier just "to be lazy." Although he/she has not been able to give it a try, Caregiver $\mathrm{H}$ is aware that he/she needs to provide incentives to encourage his/her children to start walking to school. Caregiver $\mathrm{H}$ has a vehicle in which all the family fit. However, "there is no room for picking up friends." They also have one other car in the family. Also one son has got a bike, which he used for a while but no longer does because he/she has got nowhere to leave it at school. Using the vehicle is convenient and Caregiver $\mathrm{H}$ finds himself/herself very reliant on this form of transport. Caregiver $\mathrm{H}$ considers that "when you add up all the indirect costs of walking, such as time commitments, that it is not cheap."

\subsubsection{Caregiver I}

Caregiver I describes an "important factor affecting the journey to school is what caregivers do after they have dropped their kids off at school." Caregiver I has one car in their household. He/she describes it as inconvenient taking the car because of issues of what to do with it after the journey to school. The car is infrequently used for the journey to school. One day a week Caregiver I does not work and sometimes he/she takes the car on this day if he/she needs to be somewhere soon after. The 
children have bikes available for the journey to school, but "it is far too steep to use them."

\subsubsection{Caregiver J}

One car (in which only 5 of the 6 household members can fit) is available for the journey to school. "Using the car for the journey to school is a hassle because of parking” once Caregiver J gets to work having taken the children to school.

Caregiver $\mathrm{J}$ is the only driver in the household. The cost of using the car is not the only consideration, as developing independence for the children is also considered valuable. Catching a bus is a readily available option for the journey to school and is close for both pick-up and set-down. Bikes are available for the journey to school.

\section{What Role Models do Caregivers Provide for their Children in Regards to the Journey to School?}

\subsection{WSB}

\subsubsection{Caregiver A}

Caregiver A walked to school, but "it was only about 100 metres away and it did not involve crossing any roads." Sometimes he/she walked by him/herself and sometimes with his/her cousins who lived next door. Caregiver A describes

him/herself as "not as much of a walker now as he/she would like to be." Being constrained for time restricts how much walking he/she can do. Caregiver A walks when he/she can, but his/her "work is spread around the place and it is too far to walk everywhere and [he/she] has lots of gear to cart around." Caregiver A's partner walks to work everyday. For recreation Caregiver A's family might go for a walk, but this would not just be from their house and instead they would require a ride in the car first.

\subsubsection{Caregiver $B$}

Caregiver B appears keen to actively present him/herself as a role model for good environmental habits. Caregiver B stresses that they have a very small car, which they try to fill up before they use it. Caregiver B is proud that she is a cycle commuter and mentions, "this is one step up" from his/her previous reliance on 
public transport. This stepping up the scale of environmental friendliness seems a priority. Caregiver B describes how, as a family, they use "green" transport modes as part of their recreation, for example going for a bike ride. Caregiver B's efforts at personally promoting environmentally activities underlies his/her central involvement in establishing the WSB at his/her child's school and using green modes of transport as much as possible. Caregiver B grew up in the practice of walking or cycling where possible or using public transport or car-pooling. Caregiver B walks everyday but is beginning to walk less and cycle more.

\subsubsection{Caregiver $\mathrm{C}$}

Caregiver $\mathrm{C}$ walked to school after being driven when he/she was very young. Then he/she biked to school. Caregiver $\mathrm{C}$ describes him/herself as always having been active. Caregiver $\mathrm{C}$ walks where he/she can and fitness is an important element of this. Caregiver $\mathrm{C}$ would walk more if he/she did not have kids to organise.

\subsubsection{Caregiver D}

Caregiver D walked to school and high school as well. He/she walked as part of a group. Due to an injury, walking is now Caregiver D's main form of exercise and he/she walks almost everywhere. Most of the amenities that he/she needs are within walking distance from home.

\subsection{Non-WSB}

\subsubsection{Caregiver E}

Caregiver E's partner has a large walk to work in the summer months. This walk is to work only and is on the down hill leg. When they used to walk to school from their previous house both Caregiver $\mathrm{E}$ and his/her partner took turns walking their son. Walking as a mode of transport is not used by Caregiver E. Caregiver E says that he/she does not walk unless he/she can help it. Caregiver E fits his/her walking into his/her schedule and not his/her schedule to walking. "Work schedules and pressures are very demanding" and shape other parts of Caregiver E's lifestyle. In the past when work commitments were not so constraining, Caregiver E walked more. Walking is not reinforced on the weekend either. Instead they "often go shopping and resting." 


\subsubsection{Caregiver $F$}

Walking is not very common for Caregiver F. Caregiver F "drives as much as [he/she] can if [he/she] needs to get somewhere." Catching the bus is something that is a second best when taking the car is not an option. Caregiver F does not "walk even if it's only three minutes away." Caregiver F "walked all the time when [he/she] went to school."

\subsubsection{Caregiver $\mathbf{G}$}

Caregiver $\mathrm{G}$ uses the car to get places and does not usually walk. Walking is not a priority and is not incorporated into Caregiver G's daily routine. Although Caregiver $\mathrm{G}$ tries to go for a long walk with his/her friends once a week, this does not involve his/her son and does not show walking as a way of getting around as an alternative to the car. Caregiver G walked to the same school when he/she went to school.

\subsubsection{Caregiver $H$}

Caregiver $\mathrm{H}$ was enthusiastic in his/her attitude towards walking as a method of transport. However, Caregiver $\mathrm{H}$ sees that walking also requires using public transport, which is inconvenient, especially with having a newborn child. So Caregiver $\mathrm{H}$ ends up using the car a lot. His/her situation is highly constrained due to work commitments and looking after the family. Walking is "good for health reasons and it's cheap." In Caregiver H's profession he/she promotes walking as good and healthy, but finds him/herself unable to practice this advice. Caregiver $\mathrm{H}$ also wants to encourage his/her children to walk more. Caregiver $\mathrm{H}$ sees this not just in terms of getting from place to place but as a recreation activity. In the summer Caregiver $\mathrm{H}$ describes that they go for walks down to the beach as a family. Caregiver $\mathrm{H}$ did not walk to school when he/she was young. This was despite the fact that his/her school was next door. Instead he/she got a lift with his/her mother. The walking that Caregiver $\mathrm{H}$ currently does involves helping his/her son once a week deliver his papers when he has rugby practice. Also Caregiver $\mathrm{H}$ walks around while windowshopping.

\subsubsection{Caregiver $I$}

Caregiver I prefers to walk to work and his/her route to work goes right through the school. Sometimes Caregiver I prefers to bus. Caregiver I's partner prefers walking 
to other modes of getting to work and has always done so. Caregiver I's partner does not have a car park at work and Caregiver I has car parking available only if he/she arrives really early and this does not suit. Both Caregiver I and his/her partner like to walk places and recognise this as being one of the attractions of living close to the city centre. Caregiver I has "arranged [his/her] work hours to fit walking in the journey to school." Caregiver I used to bike when she lived and went to school in the country. In the past Caregiver I's circumstances have been so constrained so he/she noted that there was no option but to drive to school. This was expensive and Caregiver I was "pleased to get out of that routine." Caregiver I tends to walk as much as possible into town, "except when getting lots of books from the library or groceries from the supermarket."

\subsubsection{Caregiver $\mathbf{J}$}

Caregiver J normally walks home from work, which takes about an hour. Having been unwell lately he/she has been unable to do this though. On the journey to work Caregiver J catches the bus. All members of the household walk to get places if things, such as the weather permit, it is within a couple of kilometres, and so long as they do not have to carry something that is too heavy. They all walk places, such as to the beach as a form of recreation. Caregiver $\mathrm{J}$ and partner walked to school when they were children.

\section{Decisions About and Involvement With the Journey to School}

\subsection{WSB}

\subsubsection{Caregiver $A$}

Caregiver A is directly involved with only part of the journey to school every morning. On two days he/she drives the WSB for the full route and on the other days he/she only takes his/her son part of the way where he meets up with the WSB, which is being driven by someone else. Caregiver $\mathrm{A}$ is the main person organising his/her son for the journey to school. Sometimes Caregiver A's partner helps. Caregiver A's son also does some things to help get himself ready. Caregiver A was 
the person in his/her household who decided about the journey to school. This was consented to anyway by his/her child who "quite likes the arrangement." However, Caregiver A suggests that he/she could have "talked [him/her] into it anyway."

\subsubsection{Caregiver B}

All three adult members of the household work together to get Caregiver B's son ready for the journey to school each morning. Caregiver $B$ is the driver of the WSB only one day per week. Caregiver B's partner drives the WSB for two days per week and another driver drives the WSB for the final two days of the school week. Within Caregiver B's household there was not any discussion about how the journey to school would occur and Caregiver B "just decided."

\subsubsection{Caregiver $\mathrm{C}$}

There are not any other potential WSB drivers available to help Caregiver C.

Caregiver $\mathrm{C}$ mentions that "it would be great if there was someone who could take the Walking School Bus five days a week." However, Caregiver C mentions that he/she would still have to take his/her kids early because he/she has to be out of the door by 7:30am and there would be no one to look after the children until the WSB came to pick them up. Caregiver $\mathrm{C}$ gets his/her children ready before they leave on the journey to school and "it is a big thing just getting them ready on time." Caregiver $\mathrm{C}$ meets his/her children for the journey home as well. Caregiver $\mathrm{C}$ walked

before the WSB was set up and "it just seems to have become [his/her] responsibility to drive it." When Caregiver C cannot take his/her children to school he/she can ring his/her sister who also has got children going to the same school and can often help out. Once a week the children's other caregiver organises getting the children to school as they stay at his/her place. Caregiver $\mathrm{C}$ made the decision about his/her children's journey's to school. This arrangement happened as this is what fitted all the constraints in Caregiver C's life.

Caregiver C's daughter who walks with the WSB does not like walking because it is too long and too hard. Caregiver $\mathrm{C}$ thinks it is hard because his/her daughter is not used to doing it regularly and it would become easier with practice. Also, sometimes Caregiver C's daughter asks to walk when this does not suit.

\subsubsection{Caregiver D}


Caregiver D is the only person available to take the WSB. As such Caregiver D has to be involved in the journey to school throughout the year. Fewer children are usually involved with the WSB on the journey home after school. Note though that about once a week Caregiver D lets his/her 8 and 11-year-old children do the journey to school by themselves. Caregiver D also picks his/her children up with the WSB in the afternoons. Caregiver D gets his/her children ready before they leave on the journey to school in the morning.

\subsection{Non-WSB}

\subsubsection{Caregiver $\mathbf{E}$}

After school both Caregiver E and partner are involved in the journey to school. This involves swapping who drives the car and picking up one person from work and dropping the other one off and then picking up their son from an after school care centre. Caregiver E takes the journey to school, unless his/her partner has time off from work. Caregiver E's partner makes their son's lunch and gets things ready the night before the journey to school. Caregiver E and his/her partner decided about the journey to school. However, it was not really a situation involving a deliberate decision making process. Caregiver $\mathrm{E}$ is aware that his/her son's occasional requests regarding the journey to school are often not possible due to constraints in their (adult) lives.

\subsubsection{Caregiver $\mathbf{F}$}

In Caregiver F's situation it is his/her family role to manage the journey to school involving both getting his/her children ready to leave the house and on the way to school. Caregiver F's partner has long hours of work commitments that prevent him/her from being involved in the journey to school. Caregiver F does not know of anyone else who would be able to help with the journey to school and about once a year he/she is in a situation where there is nobody to get the children to school so

they stay at home. After school he/she sometimes relies on wider family to help with the supervision of the journey home. However, mostly he/she meets the children.

\subsubsection{Caregiver $G$}


Caregiver $\mathrm{G}$ is directly involved in the journey to school every morning and afternoon, except for a few situations. Caregiver $\mathrm{G}$ does not know many caregivers in the school community to help supervise his/her son's journey to school. Caregiver $\mathrm{G}$ linked his/her interest in this WSB research to their own interest in developing a network of people to call on for help in getting their child to school. For the journey to school it is also important that Caregiver G's son is happy with the arrangement. However, Caregiver G says that this can be manipulated and he/she "just lets him think that [he/she] is deciding." Caregiver G plays a supervisory role in getting his/her son organised before leaving to school. Caregiver G tries to get his/her child to do as much as he can. When Caregiver $G$ cannot directly be involved in the journey to school he/she can call on help from family or a flatmate. Mostly Caregiver $\mathrm{G}$ asks people to help with his/her son's journey to school if he/she feels comfortable asking and that it will not "put the other person out." Caregiver G does not feel that his/her son would decide to do something much different to what happens currently even if he/she could.

\subsubsection{Caregiver $\mathbf{H}$}

Caregiver $\mathrm{H}$ both drops his/her children off at school and picks them up afterwards.

One day per week Caregiver $\mathrm{H}$ has got commitments after school and his/her children go to the local library until he/she can pick them up and take them home. Caregiver H's work means that he/she has to leave the house early. As there is nobody available to supervise the journey to school and the fact that he/she goes past the school anyway to drop her little children to the day care, it is easier for everyone to get in the vehicle. Furthermore, Caregiver $\mathrm{H}$ cannot leave his/her children at home by themselves to leave after him/her with the WSB. When Caregiver H cannot drop his/her children off or pick them up, his/her partner can. However, this means that he/she arrives at work late and he/she needs to stay on till later to make up for the lost time. There is just Caregiver $\mathrm{H}$ and his/her partner and no one else available for the journey to school. The older kids know what is expected of them for getting ready for the journey to school and they work together to get ready for school. Caregiver $\mathrm{H}$ takes the children to school because he/she works part-time and his/her partner works full-time. 


\subsubsection{Caregiver I}

Three days a week Caregiver I takes his/her children to school and two days a week his/her partner does it. And if neither of them are available for taking their children to school then the next-door neighbour quite often can take their children to school. There is a useful reciprocal arrangement with the neighbours who have children of similar age and who also go to the same school. Usually it is Caregiver I who meets the kids after school, alternatively the neighbours will do it. Caregiver I gets his/her children ready before the journey to school if he/she is taking them and vice versa if his/her partner is taking them. Caregiver I noted that he/her and his/her partner decided together about the journey to school. The journey to school was not really decided upon after great deliberation. Like many things in life it was more a case of just happening and they had not really considered any other methods of getting to school.

\subsubsection{Caregiver $\mathbf{J}$}

Caregiver J's two boys at primary school walk by themselves to school or occasionally they will take the bus. Caregiver J and his/her partner decided what mode of transport the children were to use when travelling to school. They talked about it and the "children are happy with the arrangement and do not complain." Caregiver $\mathrm{J}$ has an open relationship with his/her children and the children have not really asked to change the arrangement. Caregiver $\mathrm{J}$ sees that his/her children are capable of getting themselves ready for the day at school. They have been able to develop their independence. Both Caregiver $\mathbf{J}$ and his/her partner have full time work commitments.

\section{How Long does the Journey to School take and how Important is Convenience?}

\subsection{WSB}

\subsubsection{Caregiver A}

The journey to school takes up to 25 minutes. However, Caregiver A only needs to drive the WSB twice a week and for three days he/she saves about an hour. 


\subsubsection{Caregiver B}

Caregiver B describes the journey to school as taking "a surprising amount of time." It takes about 35 minutes and Caregiver B acknowledges that this long duration may mean that some children do not join because they would have to get up too early. The timing of the journey to school requires some controlling so as to be synchronised for the group.

The WSB reduces the time spend on the journey to school overall through a week because of time saved on mornings when Caregiver B does not have to drive the WSB.

\subsubsection{Caregiver $\mathbf{C}$}

When the WSB operates it takes about half and hour and Caregiver C "gets the kids walking quickly." There is only one day per week that the WSB operates and this is dependent on the weather. For the other four days they take the car as they leave half an hour earlier at 7:30am. Leaving this early is necessary so Caregiver $\mathrm{C}$ can get to work early. However, it is too early at 7:30am to take the WSB. So taking the WSB once per week is all the Caregiver $\mathrm{C}$ can afford in terms of time away from work. Walking both ways to and from school is too far, so when the WSB operates it is only for the journey to school and not after school. For the return journey they get a lift home with a relative. Caregiver $\mathrm{C}$ is highly constrained by his/her job.

\subsubsection{Caregiver D}

The journey to school takes about 10 minutes.

\subsection{Non-WSB}

\subsubsection{Caregiver $\mathbf{E}$}

Caregiver E works night shift so getting up as late as possible for the journey to school is a priority. Having to catch the bus involves getting ready almost half an hour earlier. Caregiver E is "not a morning person." The journey to school takes about 20 minutes and Caregiver E does not want to be late. However, the traffic jams often make them 10 minutes late. Taking the car for the journey to school means that Caregiver E does not have to get dressed fully. Instead he/she can just jump in the car and this reduces preparation time drastically. 


\subsubsection{Caregiver $\mathbf{F}$}

Caregiver F describes the journey to school as too far to walk and that he/she cannot afford the extra half hour to forty minutes that would be needed to walk his/her children to school. When they use the bus for the journey to school they have to leave about half an hour earlier. When they travel by car Caregiver F describes that it takes about 5-10 minutes.

\subsubsection{Caregiver $\mathbf{G}$}

The journey to school takes about five minutes. Mornings for Caregiver G are often rushed for time and he/she takes his/her son only part of the way and then watches until he is very near to school and then he/she leaves to work. In the afternoons Caregiver $\mathrm{G}$ describes enjoying exploring the environment on the way home, which takes time. Priorities for Caregiver $\mathrm{G}$ in the journey to school are timing and fitting it to his/her timetable.

\subsubsection{Caregiver $\mathbf{H}$}

Caregiver $\mathrm{H}$ used to be involved with a WSB, but due to time and work pressures and having a large family it was much easier to drop everyone off at school.

\subsubsection{Caregiver I}

The journey to school with Caregiver I's five-year-old son takes about 15-20 minutes and with just /hisher nine-year-old daughter it takes about ten minutes. "It is a little quicker coming home because it's down hill." Walking for the journey to school is convenient compared to taking the car. Convenience is the most important factor behind the journey to school.

\subsubsection{Caregiver J}

The journey to school takes about half an hour to forty minutes. The time it takes depends on whether his/her boys are fighting amongst themselves. The journey to school is constrained in that there is no convenient alternative for Caregiver $\mathrm{J}$, as other options would take too much time or money. 


\section{What are the Traffic Conditions like for the Journey to School?}

\subsection{WSB}

\subsubsection{Caregiver $\mathbf{A}$}

Caregiver A describes the traffic as sometimes being so jammed that it is doesn't move at all and when it is really busy the WSB is faster than the cars. Other times the traffic is a fairly steady stream. Traffic safety is a major factor in determining the journey to school route as opposed to alternative routes.

\subsubsection{Caregiver B}

In addition to traffic issues on the road in the journey to school, it seems that cars parked on the footpath pose a significant issue, in terms of safety and convenience. It is not uncommon to have to walk on the road in the path of oncoming traffic as a result of cars parked on the footpath. Caregiver B describes the road crossing in the journey to school as not that bad. There is a zebra crossing for the major road crossing, which Caregiver B describes as a great feature. However, people do not wait or are aggressive in respect to this feature. Caregiver B describes the road as very busy with standstill jams. In situations like this the WSB can play the game of "racing cars." Caregiver B also recognises that "the parked cars provide a safety barrier for children from cars on the road." Traffic issues seem to be part of the reason for not taking an alternative route for the journey to school.

\subsubsection{Caregiver $\mathrm{C}$}

Caregiver $\mathrm{C}$ describes that there are lots of cars around during the journey to school. Caregiver C likes being on the "main drag" as the WSB has got a higher profile here. This factor helps shape the journey to school routes as opposed to an alternative and quieter route.

\subsubsection{Caregiver D}

The journey to school involves crossing a dangerous driveway with a blind spot. Caregiver D makes the journey to school route along the main road (which does not go through busy roads anyway). This decision is to avoid quiet and potentially 
dangerous alleyways. Caregiver D sees young people using these areas and thinks that it is dangerous.

\subsection{Non-WSB}

\subsubsection{Caregiver $\mathbf{E}$}

Caregiver E describes that heaps of traffic is encountered on the way to school.

Caregiver E describes the resulting delays, as being only about 10 minutes, which he/she considers is not an issue, especially when compared to Auckland. Nonetheless Caregiver E comes close to disliking just sitting in traffic on the journey to school. A traffic issue that concerns Caregiver $\mathrm{E}$ is that pedestrians might walk out in front of $\mathrm{him} /$ her without him/her even seeing them. This is especially the case around the school.

\subsubsection{Caregiver $\mathbf{F}$}

During the journey to school in the morning the roads are busy with traffic. There are big lines of traffic and these slow the journey to school down considerably. The public bus is still on time even in peak hour traffic.

\subsubsection{Caregiver $\mathbf{G}$}

Caregiver $\mathrm{G}$ describes the one road that his/her child has to cross as quite busy and quite dangerous for kids on their own. Caregiver G describes "all the parked cars and recycling and rubbish on rubbish day clutters up the street and is something that has to be negotiated with care." The view of traffic on the road is highly concealed for children and this poses quite a danger.

\subsubsection{Caregiver $\mathbf{H}$}

Caregiver $\mathrm{H}$ describes the roads as very busy during the journey to school. Caregiver $\mathrm{H}$ singles out buses as being particularly a problem and taking up lots of room.

\subsubsection{Caregiver I}

Because Caregiver I's children are supervised during the journey to school he/she describes the traffic as being fine. However, even with a zebra crossing for the major road crossing in the journey to school Caregiver I does not like the idea of his/her kids crossing the road by themselves. Caregiver I feels that cars do not stop and there is lots of traffic, which makes it a tricky crossing. Even with the help of a zebra 
crossing to negotiate the major road, "it is quite scary" and he/she is not comfortable. One of the reasons Caregiver I does not take an alternative route to school is because of traffic issues. Caregiver I does not like the traffic on the main busy street in the journey to school. There are also dangerous entranceways across the footpath for some shops. Also cars parked on the footpath mean that you have to walk on the road, which is not good.

\subsubsection{Caregiver J}

Caregiver $\mathrm{J}$ is not overly concerned about the traffic because there are zebra crossings throughout the journey to school and his/her children use these. Caregiver $\mathbf{J}$ is aware that there is much traffic on the road along the journey to school but does not perceive this as something negative. Instead it provides a source of people able to look out for his/her children. In road safety terms the journey to school is quite safe. The busy road traffic can slow the boys down when they have to wait for cars to stop at zebra crossings.

\section{What Effect does the Weather have on the Journey to School?}

\subsection{WSB}

\subsubsection{Caregiver A}

"The weather has to be really blowing and wet" for the WSB not to be used for the journey to school. If a car is taken then all WSB members car pool together.

\subsubsection{Caregiver B}

The journey to school is not very sensitive to weather conditions. However, "if the weather is absolutely appalling" then all people involved in the WSB ring around and they all car pool together. This only happens once or twice a term though. It is necessary to avoid the children getting wet and cold.

\subsubsection{Caregiver $\mathrm{C}$}

The once-weekly WSB is stopped if it is raining. It is such a long walk that Caregiver $\mathrm{C}$ could not put the kids through it in the wet.

\subsubsection{Caregiver D}


The WSB journey to school occurs regardless of what the weather is like. Caregiver D explains that if they need to, the children put on their wet weather gear and it is not far anyway.

\subsection{Non-WSB}

\subsubsection{Caregiver $\mathbf{E}$}

The weather has no direct affect on the journey to school because inside the car it is warm and secure from the elements.

\subsubsection{Caregiver $\mathbf{F}$}

If it is sunny Caregiver F drops his/her children at a park nearby to the school through which they can walk (unsupervised) to get to school. However, if it is raining Caregiver F will drive them right up to school.

\subsubsection{Caregiver $\mathbf{G}$}

Caregiver G's son has been going to school for only five months and only a couple of times does Caregiver G remember it raining. Caregiver G's mode of journey to school is not very sensitive to weather, unless it is really wet and then he/she would drive. When it is wet it is quicker for Caregiver $\mathrm{G}$ to walk with his/her son all the way to class rather than watching from a distance as he walks the best bit by himself.

\subsubsection{Caregiver $\mathbf{H}$}

Since they no longer are involved in the WSB, the weather does not affect the journey to school as they travel by car.

\subsubsection{Caregiver I}

It would have to raining really hard for Caregiver I to consider using a car for the journey to school. Getting wet while walking to school is avoided as much as possible because it is uncomfortable.

\subsubsection{Caregiver J}

Walking for the journey to school is weather permitted. If it is fine they walk but if it is raining then they catch the bus. 